\title{
Polynuclear Complexes: Two Amino-Phenol Macrocycles Spaced by Several Linear Polyamines; Synthesis, Binding Properties, and Crystal Structure
}

\author{
Gianluca Ambrosi, ${ }^{\dagger}$ Mauro Formica, ${ }^{\dagger}$ Vieri Fusi, ${ }^{, \dagger}$ Luca Giorgi, ${ }^{\dagger}$ Eleonora Macedi, ${ }^{\dagger}$ Mauro Micheloni, ${ }^{*},{ }^{\dagger}$ \\ Paola Paoli, ${ }^{\ddagger}$ and Patrizia Rossi ${ }^{\ddagger}$ \\ ${ }^{\dagger}$ Institute of Chemical Sciences, University of Urbino, P.zza Rinascimento 6, I-61029 Urbino, Italy, and \\ *'Sergio Stecco’ Department of Energy Engineering, University of Florence, Via S. Marta 3, \\ I-50139 Florence, Italy
}

Received July 23, 2009

\begin{abstract}
The synthesis and characterization of the new polytopic ligands 1,14-bis(3,6,9-triaza-15-hydroxybicyclo[9.3.1]pentadeca$11,13,1^{15}$-trien-6-yl)-3,6,9,12-tetraazatetradecane L1, 1,15-bis(3,6,9-triaza-15-hydroxybicyclo[9.3.1]pentadeca-11,13,1 ${ }^{15}$. trien-6-yl)-3,6,10,13-tetraazapentadecane L2, and 1,16-bis(3,6,9-triaza-15-hydroxybicyclo[9.3.1]pentadeca-11,13,1 ${ }^{15}$-trien-6-yl)-3,7,10,14-tetraazahexadecane L3, containing two equal amino-phenol macrocycles spaced by several linear tetraamines, are reported. The basicity and coordination behavior toward the $\mathrm{Cu}$ (II) ion were potentiometrically determined in aqueous solution at $298.1 \mathrm{~K}$. All the ligands show similar acid-base properties behaving as octaprotic bases in the examined $\mathrm{pH}$ range ( $\mathrm{pH}=2-12$ ). The acid protons of $\mathbf{L} 1-\mathrm{L} 3$ cannot be removed under the experimental conditions used; thus, the main deprotonated species obtainable in aqueous solution are the neutral ligands, having amphionic character as demonstrated by UV - vis experiments. These species are able to form mono-, di-, and trinuclear Cu(II) complexes having stoichiometry $[\mathrm{CuL}]^{2+},\left[\mathrm{Cu}_{2} \mathrm{~L}\right]^{4+}$, and $\left[\mathrm{Cu}_{3} \mathrm{~L}\right]^{6+}$, respectively, that can lose one or two protons giving rise to $\left[\mathrm{CuH}_{-1} \mathrm{~L}\right]^{+}$, $\left[\mathrm{Cu}_{2} \mathrm{H}_{-2} \mathrm{~L}\right]^{2+}$, and $\left[\mathrm{Cu}_{3} \mathrm{H}_{-2} \mathrm{~L}\right]^{4+}$. Depending on the used ligand to metal molar ratio, the mono-, di-, or trinuclear species prevail over the others in solution. Both di- and trinuclear complexes are able to add secondary ligands (such as $\mathrm{OH}^{-}$), and in some cases two $\mathrm{Cu}(\mathrm{II})$ can cooperate to stabilize them by coordinating the guest in a bridged conformation. The structure of the $\left[\mathrm{Cu}_{2} \mathrm{~L} 3\right]^{4+}$ cation was resolved by $\mathrm{X}$-ray analysis of the $\left\{\left[\mathrm{Cu}_{2} \mathrm{~L} 3\right]\left(\mathrm{ClO}_{4}\right)_{4} \cdot 3 \mathrm{H}_{2} \mathrm{O}\right\}_{2} \cdot \mathrm{H}_{2} \mathrm{O}$ crystalline complex. It shows that each $\mathrm{Cu}(\mathrm{II})$ is penta-coordinated by one phenolate oxygen, two amine functions, belonging to one macrocyclic unit, and two amine functions of the spacer; in this species the distance between the two Cu(II) is about $5.3 \AA$.
\end{abstract}

\section{Introduction}

Ligands able to form polynuclear metal complexes are of great interest in the synthesis of metallo-receptors useful in molecular recognition, transport, and catalysis. ${ }^{1-4}$ Two requisites are fundamental to create effective and selective receptors: (i) the metal centers have to be forced by the molecular topology to stay at a fixed distance from each other

*To whom correspondence should be addressed. Tel/Fax: +39-0722350032. E-mail: vieri.fusi@uniurb.it.

(1) Lehn, J. M. Angew. Chem., Int. Ed. Engl. 1988, 27, 89.

(2) (a) Schneider, H. J.; Yatsimirsky, A. K. Principles and Methods in Supramolecular Chemistry; John Wiley \& Sons: New York, 2000. (b) Steed, J. W.; Atwood, J. L. Supramolecular Chemistry; John Wiley \& Sons: New York, 2000. (c) Voegtle, F. Comprehensive Supramolecular Chemistry: Molecular Recognition: Vol. 2, Receptors for Molecular Guests; Pergamon: Elmsford, NY, 1996. (d) Gokel, G. W. Comprehensive Supramolecular Chemistry, Volume 1: Molecular Recognition: Receptors for Cationic Guests; Pergamon: Elmsford, NY, 1996

(3) (a) Zelewsky A. Stereochemistry of Coordination Compounds; John Wiley \& Sons: New York, 1996. (b) Hancock, R. D. Metal Complexes in Aqueous Solution; Modern Inorganic Chemistry; Plenum Press: New York, 1996. (4) (a) Gokel, G. W.; Leevy, W. M.; Weber, M. E. Chem. Rev. 2004, 104 , 2723. (b) Balzani, V.; Credi A.; Venturi M. Molecular Devices and Machines: A Journey into the Nanoworld; VCH Verlagsgesellschaft Mbh: Weinheim, 2003. and (ii) the metal ions have to show an unsaturated coordination sphere. ${ }^{5}$ Selectivity and catalytic activity can be improved by the type of metal ions bound as well as by the distance between the metal centers which can cooperate (or not) in the coordination of the guest. ${ }^{6-8}$

Recently we reported about a class of polyamino-phenolic ligands derived from polyazamacrocycles containing phenolic ionophores; we established that their metal complexes may constitute an excellent base to study the molecular recognition of different substrates, such as inorganic and

(5) (a) Gavrilova, A. L.; Bosnich, B. Chem. Rev. 2004, 104, 349. (b) Guerriero, P.; Tamburini, S.; Vigato, P. A. Coord. Chem. Rev. 1995, 110, 17. (c) Bazzicalupi, C.; Bencini, A.; Fusi, V.; Giorgi, C.; Paoletti, P.; Valtancoli, B. Inorg. Chem. 1998, 37, 941. (d) Lu, Q.; Reibespiens, J. J.; Martell, A. E.; Carroll, R. I.; Clearfield, A. Inorg. Chem. 1996, 35, 7246.

(6) (a) Bazzicalupi, C.; Bencini, A.; Bianchi, A.; Fusi, V.; Garcia-España, E.; Giorgi, C.; Llinares, J. M.; Ramirez, J. A.; Valtancoli, B. Inorg. Chem. 1999, 38, 620 and references therein. (b) Bazzicalupi, C.; Bencini, A.; Bianchi, A.; Fusi, V.; Paoletti, P.; Valtancoli, B. J. Chem. Soc. Chem. Commun. 1994, 881.

(7) Koike, T.; Inoue, M.; Kimura, E.; Shiro, M. J. Am. Chem. Soc. 1996, $118,3091$.

(8) Martell A. E.; Sawier D. T. Oxygen Complexes and Oxygen Activation by Transition Metals; Plenum Press: New York, 1987. 
organic anions and neutral molecular species. ${ }^{9}$ This is mainly a result of the high stability of the related complexes and of their high solubility in water. The presence of an aromatic optical sensor group is of particular importance, because it extends the ability of the ligand to monitor the host-guest interaction.

In this context, the ligand 15-hydroxy-3,6,9-triazabicyclo[9.3.1]pentadeca-11,13, $1^{15}$-triene $\mathbf{L} \mathbf{4}^{10,11}$ (Chart1) is able to form highly unsaturated mononuclear complexes, mainly with $\mathrm{Cu}(\mathrm{II})$, having a marked tendency to form dinuclear species having a $\mathrm{L} /$ metal molar ratio of $2: 2$ to complete the coordination sphere of the metal centers. With the aim of using this promising unit to achieve new versatile polynuclear receptors, we have synthesized a series of polytopic ligands 1,14-bis(3,6,9-triaza-15-hydroxybicyclo[9.3.1]pentadeca-11, 13,1 ${ }^{15}$-trien-6-yl)-3,6,9,12-tetraazatetradecane L1, 1,15-bis(3,6,9-triaza-15-hydroxybicyclo[9.3.1]pentadeca-11,13,15 ${ }^{15}$-trien6-yl)-3,6,10,13-tetraazapentadecane $\mathbf{L 2}$, and 1,16-bis(3,6, 9-triaza-15-hydroxybicyclo[9.3.1]pentadeca-11,13, $1^{15}$-trien-6-yl)-3,7,10,14-tetraazahexadecane $\mathbf{L 3}$, containing two L4 units separated by different linear tetra-amine chains (Chart 1); the polyamine chain has the function to act both as spacer as well as coordinating moiety. In this way, the topology of the series allows the presence of three distinct coordination sites for metal ions, the two macrocyclic L4 units, and the tetra-amine chain. Ligands able to form polynuclear complexes are interesting systems which are chosen for study because they mimic the active site of many multicopper enzymes, such as laccase, ascorbate oxidase, ceruloplasmin, and others. ${ }^{12-14}$ The role exhibited by polynuclear metal centers in nature suggests that synthetic polynuclear receptors can cover a wide range of applications ranging from catalysis to molecular recognition. For example, synthetic polynuclear copper(II) complexes have been studied and recently used for the biomimetic oxidations of substrates such as tyrosine, catechols, and benzyl alcohols. ${ }^{15 a, b}$ Casella and co-workers reported on a trinuclear $\mathrm{Cu}$ (II) complex, derived from a ligand containing two chiral L-histidine residues, which exhibits biomimetic catalytic oxidation activity toward L- and D-dihydroxyphenylalanine (DOPA), with remarkable stereoselectivity. ${ }^{15 \mathrm{c}}$

(9) (a) Bazzicalupi, C.; Bencini, A.; Bianchi, A.; Cecchi, M.; Escuder, B.; Fusi, V.; Garcia-España, E.; Giorgi, C.; Luis, S. V.; Maccagni, L.; Marcellino, V.; Paoletti, P.; Valtancoli, B. J. Am. Chem. Soc. 1999, 121, 6807 and references therein. (b) Dapporto, P.; Formica, M.; Fusi, V.; Micheloni, M.; Paoli, P.; Pontellini, R.; Romani, P.; Rossi, P.; Valtancoli, B. Eur. J. Inorg. Chem. 2000, 51. (c) Aguilar, J. A.; Descalzo, A. B.; Diaz, P.; Fusi, V.; Garcia-España, E.; Luis, S. V.; Micheloni, M.; Ramirez, J. A.; Romani, P.; Soriano, C. J. Chem Soc., Perkins II 2000, 1187. (d) Dapporto, P.; Formica, M.; Fusi, V.; Giorgi, L.; Micheloni, M.; Paoli, P.; Pontellini, R.; Rossi, P. Eur. J. Inorg. Chem. 2001, 1763.

(10) (a) Fusi, V.; Llobet, A.; Mahia, J.; Micheloni, M.; Paoli, P.; Ribas, X.; Rossi, P. Eur. J. Inorg. Chem. 2002, 987. (b) Ambrosi, G.; Formica, M.; Fusi, V.; Giorgi, L.; Macedi, E.; Micheloni, M.; Pontellini, R. Inorg. Chim. Acta 2009, 362, 3709

(11) Cangiotti, M.; Formica, M.; Fusi, V.; Giorgi, L.; Micheloni, M.; Ottaviani, M. F.; Sampaolesi, S. Eur. J. Inorg. Chem. 2004, 2853.

(12) (a) Karlin, K. D. Science 1993, 261, 701. (b) Wilcox, D. E. Chem. Rev. 1996, 96, 2435. (c) Hughes, M. N. The Inorganic Chemistry of the Biological Processes; Wiley: New York, 1981.

(13) Agnus, Y. L. Copper Coordination Chemistry: Biochemical and Inorganic Perspective; Adenine Press: New York, 1983.

(14) (a) Rivera-Carrillo, M; Raptis, R. G. Abstracts of Papers, 232nd ACS National Meeting, San Francisco, CA, USA, Sept. 10-14, 2006, INOR-163; American Chemical Society: Washington, DC, 2006. (b) Funahashi Y., Fukui, K., Ozawa, T., Masuda H., Abstracts of Papers, 233rd ACS National Meeting, Chicago, IL, USA, March 25-29, 2007, INOR-137, Publisher: American Chemical Society, Washington, D. C.
Chart 1. Ligands

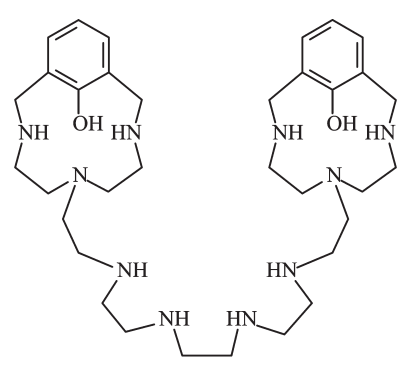

L1

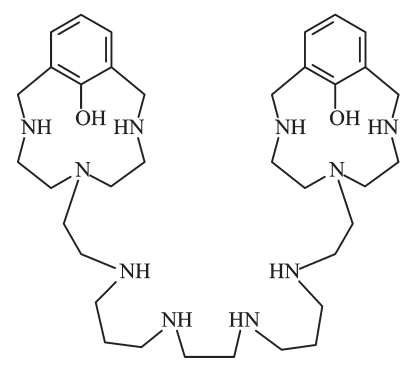

L3

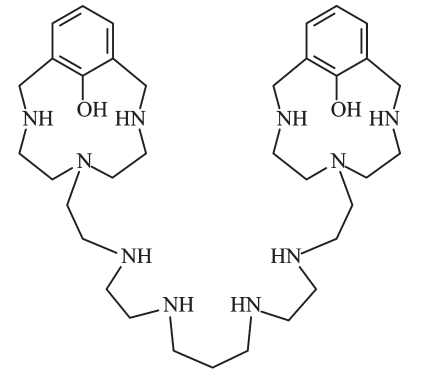

L2

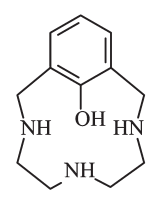

L4
Moreover, the same authors recently described the biomimetic oxidation of two catechols, namely, D- $(+)$-cathechin and $\mathrm{L}-(-)$-epicathechin, using a trinucler $\mathrm{Cu}(\mathrm{II})$ complex of a ligand based on the insertion of chiral residues derived from L-alanine. ${ }^{15 \mathrm{~d}}$ It is also to be highlighted that the trinuclear $\mathrm{Cu}(\mathrm{II})$ complexes of $\mathbf{L 1}-\mathbf{L} 3$ here reported are particularly interesting due to their water solubility; in fact, water is the ideal green solvent for many mild catalytic transformations from both economic and environmental perspectives. In this work, the synthesis of the three new ligands, together with the acid base properties and the binding behavior of the series toward the $\mathrm{Cu}$ (II) ion in aqueous solution, is reported. The crystal structure of a dinucler $\mathrm{Cu}(\mathrm{II})$ complex aids in understanding the coordination behavior of this type of ligand.

\section{Experimental Section}

General Methods. UV absorption spectra were recorded at 298 K on a Varian Cary-100 spectrophotometer equipped with a temperature control unit. IR spectra were recorded on a Shimadzu FTIR-8300 spectrometer. ${ }^{1} \mathrm{H}$ and ${ }^{13} \mathrm{C}$ NMR spectra were recorded at $298 \mathrm{~K}$ on a Bruker AVANCE-200 instrument, operating at 200.13 and $50.33 \mathrm{MHz}$, respectively. For the spectra recorded in $\mathrm{D}_{2} \mathrm{O}$, the peak positions are reported with respect to $\mathrm{HOD}(4.75 \mathrm{ppm})$ for ${ }^{1} \mathrm{H}$ NMR spectra, while dioxane was used as a reference standard in ${ }^{13} \mathrm{C}$ NMR spectra $(\delta=67.4$ ppm). For the spectra recorded in $\mathrm{CDCl}_{3}$ the peak positions are reported with respect to TMS. All reagents and solvents used were of analytical grade.

EMF Measurements. Equilibrium constants for protonation and complexation reactions of the ligands were determined by $\mathrm{pH}$-metric measurements in $0.15 \mathrm{~mol} \mathrm{dm}^{-3} \mathrm{NMe}_{4} \mathrm{Cl}$ at $298.1 \pm 0.1 \mathrm{~K}$, using the fully automatic equipment that has

(15) (a) Battaini, G.; Granata, A.; Monzani, E.; Gullotti, M.; Casella, L. Adv. Inorg. Chem. 2006, 58, 185. (b) Pawel, J. F.; Alexander, M. K.; Yauen, Y. K.; Maximilian, N. K.; Armando, J. L. P. J. Mol. Catal. A: Chem. 2009, 305, 178. (c) Santagostini, L.; Gullotti, M.; Pagliarin, R.; Monzani, E.; Casella, L Chem. Commun. 2003, 17, 2186. (d) Mutti, F. G.; Zoppellaro, G.; Gullotti, M.; Santagostini, L.; Pagliarin, R.; Andersson, K. K.; Casella, L. Eur. J. Inorg. Chem. 2009, 4, 554. 
already been described; ${ }^{16}$ the EMF data were acquired with the PASAT computer program. ${ }^{17}$ The combined glass electrode was calibrated as a hydrogen concentration probe by titrating known amounts of $\mathrm{HCl}$ with $\mathrm{CO}_{2}$-free $\mathrm{NMe}_{4} \mathrm{OH}$ solutions and determining the equivalent point by the Gran's method, ${ }^{18}$ which gives the standard potential $E^{\circ}$ and the ionic product of water $\left(\mathrm{p} K_{\mathrm{w}}=13.73(1)\right.$ at $298.1 \mathrm{~K}$ in $0.15 \mathrm{~mol} \mathrm{dm}{ }^{-3} \mathrm{NMe}_{4} \mathrm{Cl}$, $\left.K_{\mathrm{w}}=\left[\mathrm{H}^{+}\right]\left[\mathrm{OH}^{-}\right]\right)$. At least three potentiometric titrations were performed for each system in the $\mathrm{pH}$ range 2-11, and all titrations were treated either as single sets or as separate entities, for each system; no significant variations were found in the values of the determined constants. The HYPERQUAD computer program was used to process the potentiometric data. ${ }^{19}$

Crystal Data and Refinement. For $\left\{\left[\mathrm{Cu}_{2} \mathbf{L} 3\right]\left(\mathrm{ClO}_{4}\right)_{4} \cdot 3 \mathrm{H}_{2} \mathrm{O}\right\}_{2}$. $\mathrm{H}_{2} \mathrm{O}(8)$ intensity data were collected on an Oxford Diffraction Xcalibur diffractometer equipped with a CCD area detector, using $\mathrm{Cu} \mathrm{K} \alpha$ radiation (1.54108 $\AA$ ) monochromated with a graphite prism. Data were collected through the program CrysAlis $\mathrm{CCD},{ }^{20}$ and the reduction was carried on with the program CrysAlis RED. ${ }^{21}$ Absorption correction was performed with the program ABSPACK in CrysAlis RED. The structure was solved with the direct methods of the SIR $97^{22}$ package and refined by full-matrix least-squares against $F^{2}$ with the program SHELX97. ${ }^{23}$

Geometrical calculations were performed by PARST97, ${ }^{24}$ and molecular plots were produced by the program ORTEP3. ${ }^{25}$

All the non-hydrogen atoms were refined anisotropically, and all the hydrogen atoms of ligand L3 were introduced in the calculated position and refined in agreement with the coordinates of the atoms to which they are bound. Finally the hydrogen atoms of the water molecules were not found in the Fourier synthesis and were not introduced in the refinement.

Crystallographic data and refinement parameters for $\left\{\left[\mathrm{Cu}_{2} \mathbf{L} 3\right]\left(\mathrm{ClO}_{4}\right)_{4} \cdot 3 \mathrm{H}_{2} \mathrm{O}_{2} \cdot \mathrm{H}_{2} \mathrm{O}(\mathbf{8})\right.$ are reported in Table 1. In Table 2 a selection of bond lengths and angles concerning the coordination sphere is given.

Synthesis. All chemicals were purchased from Aldrich, Fluka, and Lancaster in the highest quality commercially available. 2,5Bis(bromomethyl)anysole ${ }^{26}$ (1) N,N-bis[2-(4-methylbenzensulphonyl)ethyl]ethanolamine, ${ }^{27}(\mathbf{2})$, and 1,4,7,10-tetrakis(4-methylbenzensulphonyl)-1,4,7,10-tetraazadecane ${ }^{28}$ (6a) were prepared as reported.

6-(2-Hydroxyethyl)-15-methoxyl-3,9-bis(4-methylbenzensulphonyl)-3,6,9-triazabicyclo[9.3.1]pentadeca-11,13, $1^{15}$-triene $(3)$. $\mathrm{K}_{2} \mathrm{CO}_{3}$ (46 g, $0.3 \mathrm{~mol}$ ) was added to a solution of $N, N$ bis[2-(4-methylbenzensulphonyl)ethyl]ethanolamine (2) (15 g, $0.03 \mathrm{~mol})$ in $\mathrm{CH}_{3} \mathrm{CN}\left(500 \mathrm{~cm}^{3}\right)$ under nitrogen. The reaction

(16) Dapporto, P.; Fusi, V.; Micheloni, M.; Palma, P.; Paoli, P.; Pontellini, R. Inorg. Chim. Acta 1998, 275, 168.

(17) Fontanelli, M.; Micheloni, M. I Spanish-Italian Congr. Thermodynamics of Metal Complexes, Peñiscola, June 3-6, 1990, University of Valencia: Valencia, Spain, 1990; 41.

(18) (a) Gran, G. Analyst 1952, 77, 661. (b) Rossotti, F. J.; Rossotti, H. J. Chem. Educ. 1965, 42, 375 .

(19) Gans, P.; Sabatini, A.; Vacca, A. Talanta 1996, 43, 1739

(20) Crys Alis CCD, Oxford Diffraction Ltd., Version 1.171.29.2 (release 20.01.2006 CrysAlis171.NET), Abingdon, Oxfordshire, England, 2006.

(21) Crys Alis RED, Oxford Diffraction Ltd., Version 1.171.29.2 (release 20.01.2006 CrysAlis171.NET), Abingdon, Oxfordshire, England, 2006.

(22) Altomare, A.; Cascarano, G. L.; Giacovazzo, C.; Guagliardi, A.; Moliterni, A. G.; Burla, M. C.; Polidori, G.; Camalli, M.; Spagna, R. J. Appl. Crystallogr. 1999, 32, 115.

(23) Sheldrick G. M. SHELX 97; University of Göttingen: Göttingen, Germany, 1997.

(24) Nardelli, M. J. Appl. Crystallogr. 1995, 28, 659

(25) Farrugia, L. J. J. Appl. Crystallogr. 1997, 30, 565

(26) Zazulak, W.; Chapoteau, E.; Czech, B.-P.; Kumar, A. J. Org. Chem. $1992,57,6720$.

(27) Bazzicalupi, C.; Bencini, A.; Berni, E.; Bianchi, A.; Fedi, V.; Fusi, V.; Giorgi, C.; Paoletti, P.; Valtancoli, B. Inorg. Chem. 1999, 38, 4115.

(28) Bencini, A.; Bianchi, A.; Garcia-España, E.; Giusti, M.; Mangani, S.; Micheloni, M.; Orioli, P.; Paoletti, P. Inorg. Chem. 1987, 23, 3903.
Table 1. Crystallographic Data and Refinement Parameters for $\mathbf{8}$

8

$\begin{array}{ll}\text { chemical formula } & \left\{\left[\mathrm{Cu}_{2} \mathbf{L} \mathbf{L}\right]\left(\mathrm{ClO}_{4}\right)_{4} \cdot 3 \mathrm{H}_{2} \mathrm{O}\right\}_{2} \cdot \mathrm{H}_{2} \mathrm{O} \\ M & 2513.82 \\ T(\mathrm{~K}) & 150 \\ \lambda(\AA) & 1.54180 \\ \text { cryst. syst., space group } & \text { orthorhombic, } P 2_{1} 2_{1} 2_{1} \\ \text { cell parameters }(\AA) & a=11.2076(7)^{a} \\ & b=24.643(2) \\ & c=36.939(3) \\ V\left(\AA^{3}\right) & 10202.1(13) \\ Z, d_{\mathrm{c}}\left(\mathrm{g} / \mathrm{cm}^{3}\right) & 4,1.637 \\ \mu\left(\mathrm{mm}^{-1}\right) & 3.721 \\ \text { reflections collected } / \text { unique } & 44110 / 13765[R=0.0432] \\ \text { refinement method } & \text { full-matrix least-squares on } F^{2} \\ \text { data/parameters } & 13765 / 1325 \\ \text { Final R indices }[I>2 \sigma(I)] & \mathrm{R} 1=0.0592, \mathrm{wR} 2=0.1507 \\ \mathrm{R} \text { indices (all data) } & \mathrm{R} 1=0.0821, \mathrm{wR}^{2}=0.1635\end{array}$

${ }^{a}$ Values in parentheses are the standard deviations on the last significant figure.

Table 2. Bond Lengths $(\AA)$ and Angles (deg) of the Metal Coordination Spheres in 8

\begin{tabular}{|c|c|c|}
\hline & $\mathbf{a}^{a}$ & b \\
\hline \multicolumn{3}{|c|}{ Lengths $(\AA)$} \\
\hline $\mathrm{Cu} 1-\mathrm{O} 1$ & $1.966(5)^{b}$ & $1.967(6)$ \\
\hline $\mathrm{Cu} 1-\mathrm{N} 2$ & $2.117(7)$ & $2.120(8)$ \\
\hline $\mathrm{Cu} 1-\mathrm{N} 3$ & $2.228(7)$ & $2.225(6)$ \\
\hline $\mathrm{Cu} 1-\mathrm{N} 4$ & $2.024(6)$ & $2.028(7)$ \\
\hline $\mathrm{Cu} 1-\mathrm{N} 5$ & $2.059(6)$ & $2.032(6)$ \\
\hline $\mathrm{Cu} 2-\mathrm{O} 2$ & $1.950(5)$ & $1.939(5)$ \\
\hline $\mathrm{Cu} 2-\mathrm{N} 6$ & $2.050(6)$ & $2.042(7)$ \\
\hline $\mathrm{Cu} 2-\mathrm{N} 7$ & $2.039(7)$ & $2.020(7)$ \\
\hline $\mathrm{Cu} 2-\mathrm{N} 8$ & $2.261(6)$ & $2.224(6)$ \\
\hline $\mathrm{Cu} 2-\mathrm{N} 9$ & $2.097(7)$ & $2.075(7)$ \\
\hline \multicolumn{3}{|c|}{ Angles (deg) } \\
\hline $\mathrm{N} 2-\mathrm{Cu} 1-\mathrm{O} 1$ & $97.1(2)$ & $96.2(3)$ \\
\hline $\mathrm{N} 2-\mathrm{Cu} 1-\mathrm{N} 3$ & $82.8(2)$ & $82.1(3)$ \\
\hline $\mathrm{N} 2-\mathrm{Cu} 1-\mathrm{N} 4$ & $83.2(3)$ & $83.3(3)$ \\
\hline $\mathrm{N} 2-\mathrm{Cu} 1-\mathrm{N} 5$ & $173.3(3)$ & $173.1(3)$ \\
\hline $\mathrm{N} 3-\mathrm{Cu} 1-\mathrm{O} 1$ & $89.3(2)$ & $90.3(2)$ \\
\hline $\mathrm{N} 3-\mathrm{Cu} 1-\mathrm{N} 4$ & $114.3(3)$ & $113.0(3)$ \\
\hline $\mathrm{N} 3-\mathrm{Cu} 1-\mathrm{N} 5$ & $99.7(2)$ & $99.0(2)$ \\
\hline $\mathrm{N} 4-\mathrm{Cu} 1-\mathrm{O} 1$ & $156.2(2)$ & $156.4(3)$ \\
\hline $\mathrm{N} 4-\mathrm{Cu} 1-\mathrm{N} 5$ & $90.1(2)$ & $90.0(3)$ \\
\hline $\mathrm{N} 5-\mathrm{Cu} 1-\mathrm{O} 1$ & $89.2(2)$ & $90.7(2)$ \\
\hline $\mathrm{N} 6-\mathrm{Cu} 2-\mathrm{O} 2$ & $90.0(2)$ & $88.4(3)$ \\
\hline $\mathrm{N} 6-\mathrm{Cu} 2-\mathrm{N} 7$ & $89.0(3)$ & $90.1(3)$ \\
\hline $\mathrm{N} 6-\mathrm{Cu} 2-\mathrm{N} 8$ & $99.7(2)$ & 98.3(3) \\
\hline $\mathrm{N} 6-\mathrm{Cu} 2-\mathrm{N} 9$ & $173.3(3)$ & $174.6(3)$ \\
\hline $\mathrm{N} 7-\mathrm{Cu} 2-\mathrm{O} 2$ & $157.4(3)$ & $155.9(3)$ \\
\hline $\mathrm{N} 7-\mathrm{Cu} 2-\mathrm{N} 8$ & $113.5(3)$ & $113.1(3)$ \\
\hline $\mathrm{N} 7-\mathrm{Cu} 2-\mathrm{N} 9$ & $84.0(3)$ & $84.5(3)$ \\
\hline $\mathrm{N} 8-\mathrm{Cu} 2-\mathrm{O} 2$ & $88.9(2)$ & $90.9(2)$ \\
\hline $\mathrm{N} 8-\mathrm{Cu} 2-\mathrm{N} 9$ & $83.2(3)$ & $83.7(3)$ \\
\hline $\mathrm{N} 9-\mathrm{Cu} 2-\mathrm{O} 2$ & $96.2(2)$ & $96.7(2)$ \\
\hline
\end{tabular}

${ }^{a}$ The $\mathbf{a}$ and $\mathbf{b}$ labels refer to the two independent $\left[\mathrm{Cu}_{2} \mathbf{L} 3\right]$ $\left(\mathrm{ClO}_{4}\right]_{4} \cdot 3\left(\mathrm{H}_{2} \mathrm{O}\right)$ moieties found in the asymmetric unit. ${ }^{b}$ Values in parentheses are the standard deviations on the last significant figure.

mixture was heated to reflux, and afterward a solution of 2,5bis[2-(chloromethyl)anysole (1) $(9.7 \mathrm{~g}, 0.03 \mathrm{~mol})$ in $250 \mathrm{~cm}^{3}$ of $\mathrm{CH}_{3} \mathrm{CN}$ was added dropwise. The progress of the reaction was followed by TLC on alumina plates using $\mathrm{CHCl}_{3}$. The reaction mixture was stirred for one night and then cooled. The solvent was removed under reduced pressure, and the solid residue was treated with $\mathrm{CHCl}_{3}$, filtered, and evaporated under reduced 
pressure. The solid residue was suspended in hot $\mathrm{CH}_{3} \mathrm{CN}$, the solution was then cooled, and the solid obtained was filtered and washed with a little $\mathrm{CH}_{3} \mathrm{CN}$ and diethyl ether, to give 3 (14.2 g, $81 \%$ ). The product was recrystallized from hot $\mathrm{CH}_{3} \mathrm{CN} .{ }^{1} \mathrm{H}$ $\operatorname{NMR}\left(\mathrm{CDCl}_{3}\right) \delta=7.73(4 \mathrm{H}, \mathrm{d}, J=8.1 \mathrm{~Hz}), 7.36(4 \mathrm{H}, \mathrm{d}, J=$ $8.1 \mathrm{~Hz}), 7.30(2 \mathrm{H}, \mathrm{d}, J=8.2 \mathrm{~Hz}), 7.16\left(1 \mathrm{H}, \mathrm{dd}, J_{1}=8.2 \mathrm{~Hz}, J_{2}=\right.$ $6.4 \mathrm{~Hz}), 4.31(4 \mathrm{H}, \mathrm{s}), 3.66(3 \mathrm{H}, \mathrm{s}), 3.39(2 \mathrm{H}, \mathrm{br}), 2.99(2 \mathrm{H}, \mathrm{m})$, $2.85(2 \mathrm{H}, \mathrm{m}), 2.55(2 \mathrm{H}, \mathrm{m}), 2.50(6 \mathrm{H}, \mathrm{s}), 2.37(2 \mathrm{H}, \mathrm{t}, J=4.51)$, $1.82(2 \mathrm{H}, \mathrm{br}) ;{ }^{13} \mathrm{C} \mathrm{NMR}\left(\mathrm{CDCl}_{3}\right) \delta=158.3,143.5,135.7,133.1$, $129.9,129.2,127.1,126.0,63.2,58.5,56.6,53.2,48.0,44.7,21.6$ ppm. Anal. Calcd for $\mathrm{C}_{29} \mathrm{H}_{37} \mathrm{~N}_{3} \mathrm{O}_{6} \mathrm{~S}_{2}$ (587.8): C, 59.26; H, 6.35; N, 7.15. Found: C, 59.4; H, 6.7; N, 7.0.

6-(2-Chloroethyl)-15-methoxyl-3,9-bis(4-methylbenzensulphonyl)-3,6,9-triazabicyclo[9.3.1]pentadeca-11,13,1 ${ }^{15}$-triene (4). A solution of mesyl chloride $\left(2 \mathrm{~g}, 0.017 \mathrm{~mol}, 1.4 \mathrm{~cm}^{3}\right)$ in dry $\mathrm{CH}_{2} \mathrm{Cl}_{2}\left(100 \mathrm{~cm}^{3}\right)$ was added dropwise to a solution in an ice bath of $3(5 \mathrm{~g}, 0.0085 \mathrm{~mol})$ and TEA $\left(8.6 \mathrm{~g}, 0.085 \mathrm{~mol}, 12 \mathrm{~cm}^{3}\right)$ in dry $\mathrm{CH}_{2} \mathrm{Cl}_{2}\left(250 \mathrm{~cm}^{3}\right)$ under nitrogen. The progress of the reaction was followed by TLC on alumina plates using $\mathrm{CHCl}_{3}$. The reaction mixture was stirred for one night and then washed with $5 \% \mathrm{HCl}\left(3 \times 100 \mathrm{~cm}^{3}\right)$, aqueous $\mathrm{Na}_{2} \mathrm{CO}_{3}$ solution $(3 \times$ $\left.100 \mathrm{~cm}^{3}\right)$, aqueous $\mathrm{NaCl}$ solution $\left(1 \times 100 \mathrm{~cm}^{3}\right)$, and water $(1 \times$ $\left.100 \mathrm{~cm}^{3}\right)$. The organic solution was dried over anhydrous $\mathrm{Na}_{2} \mathrm{SO}_{4}$ and then evaporated, affording 4 (5.1 g, 98\%). ${ }^{1} \mathrm{H}$ $\operatorname{NMR}\left(\mathrm{CDCl}_{3}\right) \delta=7.74(4 \mathrm{H}, \mathrm{d}, J=8.3 \mathrm{~Hz}), 7.36(4 \mathrm{H}, \mathrm{d}, J=$ $8.3 \mathrm{~Hz}), 7.30(2 \mathrm{H}, \mathrm{d}, J=7.1 \mathrm{~Hz}), 7.17\left(1 \mathrm{H}, \mathrm{dd}, J_{1}=8.7 \mathrm{~Hz}, J_{2}=\right.$ $7.1 \mathrm{~Hz}), 4.40(2 \mathrm{H}, \mathrm{d}, J=12.2 \mathrm{~Hz}), 4.22(2 \mathrm{H}, \mathrm{d}, J=12.2 \mathrm{~Hz})$, $3.61(3 \mathrm{H}, \mathrm{s}), 3.30(2 \mathrm{H}, \mathrm{t}, J=6.9 \mathrm{~Hz}), 2.90(4 \mathrm{H}, \mathrm{m}), 2.62(4 \mathrm{H}, \mathrm{m})$, $2.46(6 \mathrm{H}, \mathrm{s}), 1.69(2 \mathrm{H}, \mathrm{br}) ;{ }^{13} \mathrm{C} \mathrm{NMR}\left(\mathrm{CDCl}_{3}\right) \delta=158.4,143.4$, 135.9, 133.2, 129.9, 129.0, 127.1, 126.1, 63.3, 57.2, 53.5, 47.4, 44.4, 42.3, $21.6 \mathrm{ppm}$. Anal. Calcd for $\mathrm{C}_{29} \mathrm{H}_{36} \mathrm{ClN}_{3} \mathrm{O}_{5} \mathrm{~S}_{2}$ (606.2): C, 57.46; H, 5.99; N, 6.93. Found: C, 57.2; H, 6.2; N, 7.1.

1,4,8,11-Tetrakis(4-methylbenzensulphonyl)-1,4,8,11-tetraazahendecane (6b). A solution of tosylchloride (27 g, $0.14 \mathrm{~mol})$ in pyridine $\left(100 \mathrm{~cm}^{3}\right)$ was heated at $313 \mathrm{~K}$, and then a solution of $N$, $N^{\prime}$-bis(2-aminoethyl)-1,3-propylendiamine (5a) (5.0 g, $0.03 \mathrm{~mol}$ ) in pyridine $\left(100 \mathrm{~cm}^{3}\right)$ was added dropwise. The reaction mixture was stirred for $2 \mathrm{~h}$ at $323 \mathrm{~K}$ and then cooled to rt. The solution was precipitated by dripping it into a water/ice mixture, which was decanted to afford a solid residue that was purified by flash chromatography $\left(\mathrm{Al}_{2} \mathrm{O}_{3}, \mathrm{CH}_{2} \mathrm{Cl}_{2} / \mathrm{CHCl}_{3} \mathrm{l}: 1\right)$, to yield $\mathbf{6 b}(19.5$ $\mathrm{g}, 81 \%) .{ }^{1} \mathrm{H}$ NMR $\left(\mathrm{CDCl}_{3}\right) \delta=7.74(4 \mathrm{H}, \mathrm{d}, J=6.7 \mathrm{~Hz}), 7.63$ $(4 \mathrm{H}, \mathrm{d}, J=8.3 \mathrm{~Hz}), 7.29(4 \mathrm{H}, \mathrm{d}, J=6.7 \mathrm{~Hz}), 7.27(4 \mathrm{H}, \mathrm{d}, J=$ $8.3 \mathrm{~Hz}), 3.15(8 \mathrm{H}, \mathrm{s}), 3.10(4 \mathrm{H}, \mathrm{t}, J=6.6 \mathrm{~Hz}), 2.41(6 \mathrm{H}, \mathrm{s}), 2.39$ $(6 \mathrm{H}, \mathrm{s}), 1.93(2 \mathrm{H}$, quintet, $J=6.6 \mathrm{~Hz}) ;{ }^{13} \mathrm{C} \mathrm{NMR}\left(\mathrm{CDCl}_{3}\right) \delta=$ $143.8,143.4,136.8,134.8,129.9,129.8,127.3,127.1,49.8,48.3$, 43.2, 28.4, 21.5 ppm. Anal. Calcd for $\mathrm{C}_{35} \mathrm{H}_{44} \mathrm{~N}_{4} \mathrm{O}_{8} \mathrm{~S}_{4}$ (777.0): C, 54.10; H, 5.71; N, 7.21. Found: C, 53.8; H, 5.9; N, 7.1

1,5,8,12-Tetrakis(4-methylbenzensulphonyl)-1,5,8,12-tetraazadodecane (6c). A solution of tosylchloride ( $86 \mathrm{~g}, 0.45 \mathrm{~mol})$ in pyridine $\left(200 \mathrm{~cm}^{3}\right)$ was heated at $313 \mathrm{~K}$, then a solution of $N, N^{\prime}$ bis(3-aminopropyl)ethylendiamine $(\mathbf{5 c})(17.5 \mathrm{~g}, 0.1 \mathrm{~mol})$ in pyridine $\left(100 \mathrm{~cm}^{3}\right)$ was added dropwise. The reaction mixture was stirred for $2 \mathrm{~h}$ at $323.1 \mathrm{~K}$ and then cooled down to rt. The solution was precipitated by dripping it into a water/ice mixture that was decanted to afford a solid residue that was treated with $\mathrm{CHCl}_{3}$ and crystallized by EtOH/ $\mathrm{CHCl}_{3}(4: 1)$, to yield $\mathbf{6 c}(43.83$ g, 55\%). ${ }^{1} \mathrm{H}$ NMR $\left(\mathrm{CDCl}_{3}\right) \delta=7.74(4 \mathrm{H}, \mathrm{d}, J=8.1 \mathrm{~Hz}), 7.67$ $(4 \mathrm{H}, \mathrm{d}, J=8.2 \mathrm{~Hz}), 7.33(4 \mathrm{H}, \mathrm{d}, J=8.1 \mathrm{~Hz}), 7.30(4 \mathrm{H}, \mathrm{d}, J=$ $8.2 \mathrm{~Hz}), 5.34(2 \mathrm{H}, \mathrm{t}, J=6.3 \mathrm{~Hz}), 3.23(4 \mathrm{H}, \mathrm{s}), 3.16(4 \mathrm{H}, \mathrm{t}, J=6.9$ $\mathrm{Hz}), 2.98(4 \mathrm{H}, \mathrm{m}), 2.45(6 \mathrm{H}, \mathrm{s}), 2.42(6 \mathrm{H}, \mathrm{s}), 1.80$ (4H, quintet, $J=6.1 \mathrm{~Hz}) ;{ }^{13} \mathrm{C} \mathrm{NMR}\left(\mathrm{CDCl}_{3}\right) \delta=143.9,143.4,136.7,135.0$, 130.0, 129.8, 127.2, 127.0, 48.9, 47.2, 40.0, 28.9, 21.5 ppm. Anal. Calcd for $\mathrm{C}_{36} \mathrm{H}_{46} \mathrm{~N}_{4} \mathrm{O}_{8} \mathrm{~S}_{4}$ (790.2): C, 54.66; H, 5.86; N, 7.08. Found: C, 54.4; H, 6.0; N, 6.9.

1,14-Bis[3,9-bis(4-methylbenzensulphonyl)-3,6,9-triaza-15-methoxybicyclo[9.3.1]pentadeca-11,13, ${ }^{15}$-trien-6-yl]- 3,6,9,12-tetrakis(4-methylbenzensulphonyl)-3,6,9,12-tetraazatetradecane (7a). A solution of $6 \mathrm{a}(2.04 \mathrm{~g}, 0.0027 \mathrm{~mol})$ and $\mathrm{K}_{2} \mathrm{CO}_{3}(7.5 \mathrm{~g}, 0.054$ mol) in $\mathrm{CH}_{3} \mathrm{CN}\left(300 \mathrm{~cm}^{3}\right)$ under nitrogen was heated to reflux, and then a solution of $4(3.3 \mathrm{~g}, 0.0054 \mathrm{~mol})$ in $\mathrm{CH}_{3} \mathrm{CN}\left(250 \mathrm{~cm}^{3}\right)$ was added. The reaction was completed in $4 \mathrm{~h}$ (TLC alumina plates $/ \mathrm{CHCl}_{3}$ ) and then cooled down and evaporated. The solid residue was treated with $\mathrm{CHCl}_{3}$ and filtered, and the solvent was removed under reduced pressure. The crude product was purified by chromatography on activated alumina $\left(8 \% \mathrm{H}_{2} \mathrm{O}\right)$ eluted with $\mathrm{CH}_{2} \mathrm{Cl}_{2}$. The obtained product was further purified by crystallization from $\mathrm{CH}_{3} \mathrm{CN} / \mathrm{EtOH}$, affording $7 \mathbf{a}(2.5 \mathrm{~g}, 49 \%)$. ${ }^{1} \mathrm{H}$ NMR $\left(\mathrm{CDCl}_{3}\right) \delta=7.79-7.73(16 \mathrm{H}, \mathrm{m}), 7.35-7.26(16 \mathrm{H}$, $\mathrm{m}), 7.24(4 \mathrm{H}, \mathrm{d}, J=7.7 \mathrm{~Hz}), 7.11\left(2 \mathrm{H}, \mathrm{dd}, J_{1}=7.7 \mathrm{~Hz}, J_{2}=\right.$ $6.50 \mathrm{~Hz}), 4.30(8 \mathrm{H}, \mathrm{br}), 3.63(6 \mathrm{H}, \mathrm{s}), 3.31$ (4H, br), $3.25(8 \mathrm{H}, \mathrm{br})$, $3.00-2.82(12 \mathrm{H}, \mathrm{m}), 2.63-2.50(6 \mathrm{H}, \mathrm{m}), 2.43(12 \mathrm{H}, \mathrm{s}), 2.41(6 \mathrm{H}$, s), $2.40(6 \mathrm{H}, \mathrm{s}), 1.74(6 \mathrm{H}, \mathrm{br}) ;{ }^{13} \mathrm{C} \mathrm{NMR}\left(\mathrm{CDCl}_{3}\right) \delta=158.4$, $143.7,143.4,143.3,135.9,135.7,135.0,133.1,130.1,129.9$, $129.9,129.0,127.5,127.4,127.2,125.9,63.1,54.0,52.3,49.7$, 49.3, 49.0, 48.6, 47.7, 43.6, 21.5 ppm. Anal. Calcd for $\mathrm{C}_{92} \mathrm{H}_{112} \mathrm{~N}_{10} \mathrm{O}_{18} \mathrm{~S}_{8}$ (1902.4): C, 58.08; H, 5.93; N, 7.36. Found: C, $57.7 ; \mathrm{H}, 6.3 ; \mathrm{N}, 7.1$.

1,15-Bis[3,9-bis(4-methylbenzensulphonyl)-3,6,9-triaza-15hydroxybicyclo[9.3.1] pentadeca-11,13, ${ }^{15}$-trien-6-yl]- 3,6,10,13tetrakis(4-methylbenzensulphonyl)-3,6,10,13-tetraazapentadecane $(\mathbf{7 b})$. This compound was obtained following the same procedure reported for $7 \mathbf{a}$ using $\mathbf{6 b}(3.03 \mathrm{~g}, 0.0039 \mathrm{~mol}), \mathrm{K}_{2} \mathrm{CO}_{3}$ $(11.0 \mathrm{~g}, 0.078 \mathrm{~mol})$, and $4(4.8 \mathrm{~g}, 0.0078 \mathrm{~mol})$, affording $3.8 \mathrm{~g}$ of 7b $(51 \%) .{ }^{1} \mathrm{H}$ NMR $\left(\mathrm{CDCl}_{3}\right) \delta=7.79-7.66(16 \mathrm{H}, \mathrm{m}), 7.35-$ $7.22(20 \mathrm{H}, \mathrm{m}), 7.12(2 \mathrm{H}, \mathrm{t}, J=7.5 \mathrm{~Hz}), 4.30(8 \mathrm{H}, \mathrm{br}), 3.60(6 \mathrm{H}$, s), 3.23-2.82 (24H, m, br), $2.56(8 \mathrm{H}, \mathrm{m}, \mathrm{br}), 2.39$ (18H, s, br), $2.36(6 \mathrm{H}, \mathrm{s}), 11.90(2 \mathrm{H}, \mathrm{m}, \mathrm{br}), 1.73(4 \mathrm{H}, \mathrm{m}, \mathrm{br}) ;{ }^{13} \mathrm{C} \mathrm{NMR}$ $\left(\mathrm{CDCl}_{3}\right) \delta=158.4,143.6,143.4,143.3,135.9,135.7,135.4$, 133.1, 129.9, 129.8, 129.0, 127.3, 127.1, 125.9, 63.2, 54.1, 52.5, 49.5, 49.1, 48.6, 47.9, 47.7, 43.7, 21.5 ppm. Anal. Calcd for $\mathrm{C}_{93} \mathrm{H}_{114} \mathrm{~N}_{10} \mathrm{O}_{18} \mathrm{~S}_{8}$ (1916.5): C, 58.29; H, 6.00; N, 7.31. Found: C, 58.1; H, 6.3; N, 7.0.

1,16-Bis[3,9-bis(4-methylbenzensulphonyl)-3,6,9-triaza-15methoxybicyclo[9.3.1]pentadeca-11,13,1 ${ }^{15}$-trien-6-yl] 3,7,10,14tetrakis(4-methylbenzensulphonyl)-3,7,10,14-tetraazatehexadecane $(\mathbf{7 c})$. This compound was obtained following the same procedure reported for $7 \mathbf{a}$ using $\mathbf{6 c}(1.7 \mathrm{~g}, 2.15 \mathrm{mmol}), \mathrm{K}_{2} \mathrm{CO}_{3}(6$ g, $43 \mathrm{mmol}$ ) and 4 (2.6 g, $4.3 \mathrm{mmol})$, affording $2.6 \mathrm{~g}$ of $7 \mathrm{c}(73 \%)$. ${ }^{\mathrm{P}} \mathrm{H}$ NMR $\left(\mathrm{CDCl}_{3}\right) \delta=7.78-7.69(16 \mathrm{H}, \mathrm{m}), 7.35-7.31(16 \mathrm{H}$, $\mathrm{m}), 7.22(4 \mathrm{H}, \mathrm{d}, J=6.7 \mathrm{~Hz}), 7.11\left(2 \mathrm{H}, \mathrm{dd}, J_{1}=8.0 \mathrm{~Hz}, J_{2}=6.8\right.$ $\mathrm{Hz}), 4.29$ (8H, br), $3.62(6 \mathrm{H}, \mathrm{s}), 3.30(4 \mathrm{H}, \mathrm{br}), 3.10(8 \mathrm{H}, \mathrm{br})$, $3.00-2.78(12 \mathrm{H}, \mathrm{m}), 2.65-2.53(6 \mathrm{H}, \mathrm{m}), 2.43(12 \mathrm{H}, \mathrm{s}), 2.40$ $(12 \mathrm{H}, \mathrm{s}), 1.81(4 \mathrm{H}, \mathrm{m}), 1.72(6 \mathrm{H}, \mathrm{br}) ;{ }^{13} \mathrm{C} \mathrm{NMR}\left(\mathrm{CDCl}_{3}\right) \delta=$ $158.4,143.6,143.4,143.3,136.0,135.9,135.3,133.1,130.0$, $129.9,129.8,128.9,127.3,127.3,127.1,126.0,63.2,54.2,53.6$, $52.2,49.4,48.1,47.7,47.4,43.6,28.5,21.5 \mathrm{ppm}$. Anal. Calcd for $\mathrm{C}_{94} \mathrm{H}_{116} \mathrm{~N}_{10} \mathrm{O}_{18} \mathrm{~S}_{8}$ (1930.5): C, 58.48; H, 6.06; N, 7.26. Found: $\mathrm{C}$, 58.2; H, 6.4; N, 7.0.

1,14-Bis(3,6,9-triaza-15-methoxybicyclo[9.3.1]pentadeca-11, $13,1^{15}$-trien-6-yl)-3,6,9,12-tetraazatetradecane (L1). On a suspension of $7 \mathbf{a}(2.2 \mathrm{~g}, 1.15 \mathrm{mmol})$ in diethyl ether $\left(30 \mathrm{~cm}^{3}\right)$ and $\mathrm{MeOH}\left(1.5 \mathrm{~cm}^{3}\right)$, cooled at $203 \mathrm{~K}$, ammonia $\left(300 \mathrm{~cm}^{3}\right)$ was condensed. Small pieces of lithium were added to the mixture until the suspension became blue. After the solution remained blue for $30 \mathrm{~min}, \mathrm{NH}_{4} \mathrm{Cl}(6 \mathrm{~g}, 0.1 \mathrm{~mol})$ was added. The solid obtained after the evaporation of the solvents was treated with 4 mol dm ${ }^{-3} \mathrm{HCl}\left(300 \mathrm{~cm}^{3}\right)$ and the resulting solution washed with $\mathrm{CHCl}_{3}\left(3 \times 100 \mathrm{~cm}^{3}\right)$. The acidic solution was filtered and then evaporated to dryness. The resultant solid was dissolved in the minimum amount of water and the solution made alkaline with concentrated $\mathrm{NaOH}$. The liquid was extracted with $\mathrm{CHCl}_{3}(3 \times$ $100 \mathrm{~cm}^{3}$ ). The organic part was dried over $\mathrm{Na}_{2} \mathrm{SO}_{4}$ and vacuum evaporated to obtain a solid that was dissolved in ethanol, filtered, and treated with a $\mathrm{HCl}$ ethanol solution until complete precipitation of a solid that was filtered off to achieve $\mathbf{L 1}$ as a hydrochloride salt $(1 \mathrm{~g}, 91 \%)$. The product was recrystallized from $\mathrm{MeOH} / \mathrm{EtOH} .{ }^{1} \mathrm{H}$ NMR $\left(\mathrm{D}_{2} \mathrm{O}\right) \delta=7.40(4 \mathrm{H}, \mathrm{d}, J=7.5$ 
$\mathrm{Hz}), 7.10(2 \mathrm{H}, \mathrm{t}, J=7.5 \mathrm{~Hz}), 4.75(4 \mathrm{H}, \mathrm{d}, J=13.6 \mathrm{~Hz}), 3.98$ $(4 \mathrm{H}, \mathrm{d}, J=13.6 \mathrm{~Hz}), 3.52(4 \mathrm{H}, \mathrm{s}), 3.45-3.23(12 \mathrm{H}, \mathrm{m}), 3.10(4 \mathrm{H}$, t, br), 2.88-2.70 (8H, m), 2.52-2.39 (4H, m), 1.77 (4H, m) ppm; ${ }^{13} \mathrm{C}$ NMR $\left(\mathrm{D}_{2} \mathrm{O}\right) \delta=153.0,133.5,124.1,123.1,48.4,47.9,46.8$, $43.5,43.3,43.2,43.0,42.8 \mathrm{ppm}$. Anal. Calcd for $\mathrm{C}_{34} \mathrm{H}_{73^{-}}$ $\mathrm{Cl}_{9} \mathrm{~N}_{10} \mathrm{O}_{4}\left(\mathbf{L 1} \cdot 9 \mathrm{HCl} \cdot 2 \mathrm{H}_{2} \mathrm{O}, 1005.1\right): \mathrm{C}, 40.63 ; \mathrm{H}, 7.32 ; \mathrm{N}$, 13.94. Found: C, 40.8; H, 7.5; N, 13.8 .

1,15-Bis(3,6,9-triaza-15-hydroxybicyclo[9.3.1]pentadeca-11, 13, $1^{15}$-trien-6-yl)-3,6,10,13-tetraazapentadecane (L2). This compound was obtained following the same procedure reported for $\mathbf{L} 1$ by using $\mathbf{7 b}$ ( $3 \mathrm{~g}, 1.6 \mathrm{mmol}$ ), affording $\mathbf{L} 2$ as its hydrochloride salt $(1.32 \mathrm{~g}, 80) .{ }^{1} \mathrm{H}$ NMR $\left(\mathrm{D}_{2} \mathrm{O}\right) \delta=7.36(4 \mathrm{H}, \mathrm{d}, J=7.6 \mathrm{~Hz})$, $7.06(2 \mathrm{H}, \mathrm{t}, J=7.6 \mathrm{~Hz}), 4.71(4 \mathrm{H}, \mathrm{d}, J=13.6 \mathrm{~Hz}), 3.94(4 \mathrm{H}, \mathrm{d}$, $J=13.6 \mathrm{~Hz}), 3.34(4 \mathrm{H}, \mathrm{s}), 3.27-3.04(16 \mathrm{H}, \mathrm{m}), 2.85-2.70(8 \mathrm{H}$, $\mathrm{m}), 2.46-2.38(4 \mathrm{H}, \mathrm{m}), 2.02(2 \mathrm{H}$, quintet), $1.77-1.70(4 \mathrm{H}, \mathrm{m})$; ${ }^{13} \mathrm{C}$ NMR $\left(\mathrm{D}_{2} \mathrm{O}\right) \delta=153.1,133.5,124.1,123.2,49.2,48.5,47.9$, 46.6, 44.9, 43.3, 43.1, 42.8, $22.6 \mathrm{ppm}$. Anal. Calcd for $\mathrm{C}_{35} \mathrm{H}_{75} \mathrm{Cl}_{9} \mathrm{~N}_{10} \mathrm{O}_{4}\left(\mathbf{L 2} \cdot 9 \mathrm{HCl} \cdot 2 \mathrm{H}_{2} \mathrm{O}, 1019.8\right): \mathrm{C}, 41.22 ; \mathrm{H}, 7.41$; N, 13.73. Found: C, 41.5; H, 7.6; N, 13.9.

1,16-Bis(3,6,9-triaza-15-methoxybicyclo[9.3.1]pentadeca-11, $13,1^{15}$-trien-6-yl)-3,7,10,14-tetraazatehexadecane ( $\left.\mathrm{L} 3\right)$. This compound was obtained following the same procedure reported for $\mathbf{L} 1$ by using $7 \mathbf{c}(3 \mathrm{~g}, 1.6 \mathrm{mmol})$, affording $\mathbf{L 3}$ as its hydrochloride salt $(1.32 \mathrm{~g}, 80 \%)$. ${ }^{1} \mathrm{H}$ NMR $\left(\mathrm{D}_{2} \mathrm{O}\right) \delta=7.38(4 \mathrm{H}, \mathrm{d}, J=7.7 \mathrm{~Hz})$, $7.09(2 \mathrm{H}, \mathrm{t}, J=7.7 \mathrm{~Hz}), 4.74(4 \mathrm{H}, \mathrm{d}, J=13.8 \mathrm{~Hz}), 3.96(4 \mathrm{H}, \mathrm{d}$, $J=13.8 \mathrm{~Hz}), 3.35(4 \mathrm{H}, \mathrm{s}), 3.30-3.05(16 \mathrm{H}, \mathrm{m}), 2.82-2.70(8 \mathrm{H}$, m), 2.46-2.39 (4H, m), 2.03 (4H, quintet), 1.79-1.72 (4H, m); ${ }^{13} \mathrm{C}$ NMR $\left(\mathrm{D}_{2} \mathrm{O}\right) \delta=153.2,133.4,123.9,123.2,48.5,47.9,46.6$, 45.0, 43.0, 42.1, 22.6 ppm. Anal. Calcd for $\mathrm{C}_{36} \mathrm{H}_{77} \mathrm{Cl}_{9} \mathrm{~N}_{10} \mathrm{O}_{4}$ $\left(\mathbf{L 3} \cdot 9 \mathrm{HCl} \cdot 2 \mathrm{H}_{2} \mathrm{O}, 1033.2\right)$ : C, 41.85; H, 7.51; N, 13.56. Found: C, 42.0; H, 7.7; N, 13.4 .

$\left\{\left[\mathrm{Cu}_{2} \mathbf{L} 3\right]\left(\mathrm{ClO}_{\mathbf{4}}\right)_{\mathbf{4}} \cdot \mathbf{3} \mathbf{H}_{\mathbf{2}} \mathrm{O}\right\}_{\mathbf{2}} \cdot \mathbf{H}_{\mathbf{2}} \mathrm{O}(\mathbf{8})$. A sample of $\mathrm{Cu}\left(\mathrm{ClO}_{4}\right)_{2}$. $6 \mathrm{H}_{2} \mathrm{O}(37 \mathrm{mg}, 0.1 \mathrm{mmol})$ in water $\left(15 \mathrm{~cm}^{3}\right)$ was added to an aqueous solution $\left(15 \mathrm{~cm}^{3}\right)$ containing $\mathbf{L 3} \cdot 9 \mathrm{HCl} \cdot 2 \mathrm{H}_{2} \mathrm{O}(54 \mathrm{mg}$, $0.05 \mathrm{mmol}$ ). The $\mathrm{pH}$ of the resulting solution was adjusted to 7 with $0.1 \mathrm{M} \mathrm{NaOH}$ and saturated with solid $\mathrm{NaClO}_{4}$. After a few minutes, 8 precipitated as a microcrystalline green solid (53 mg, 84\%). Anal. Calcd for $\mathrm{C}_{72} \mathrm{H}_{142} \mathrm{Cl}_{8} \mathrm{Cu}_{4} \mathrm{~N}_{20} \mathrm{O}_{43}$ : C, 34.40; H, 5.69; N, 11.14. Found: C, 34.2; H, 6.0; N, 11.1. Crystals suitable for X-ray analysis were obtained by slow evaporation of an aqueous solution containing $\mathbf{8}$.

\section{Results and Discussion}

Synthesis. Scheme 1 reports the synthesis of the new ligands $\mathbf{L 1}, \mathbf{L 2}$, and $\mathbf{L} \mathbf{3}$ which is based on the coupling of the macrocyclic fragment $\mathbf{4}$ with the protected polyamines $\mathbf{6 a}, \mathbf{6 b}$, and $\mathbf{6 c}$, respectively, followed by the removal of the amine and phenol protective groups.

The 6-(2-chloroethyl)-15-methoxyl-3,9-bis(4-methylbenzensulphonyl)-3,6,9-triazabicyclo[9.3.1]-pentadeca-11, $13,1^{15}$-triene 4 was prepared using a modification of the Richman-Atkins method, ${ }^{29}$ involving the cyclization of the $N, N$-bis[2-(4-methylbenzensulphonyl)ethyl]ethanolamine 2 with 1 equiv of 2,5-bis[2-(chloromethyl)anysole 1 , in the presence of an alkaline carbonate base. The final compound 6-(2-hydroxyethyl)-15-methoxyl-3,9-bis(4-methylbenzensulphonyl)-3,6,9-triazabicyclo[9.3.1]pentadeca- $11,13,1^{15}$-triene 3 was obtained by a $1+1$ cyclization scheme, and it was obtained from the crude product by recrystallization from acetonitrile. Compound $\mathbf{4}$ was obtained by mild chlorination of $\mathbf{3}$ with mesyl chloride in dichloromethane and triethylamine; this step did not require any further purification process.

Full protected ligands $7 \mathbf{a}, 7 \mathbf{7 b}$, and $\mathbf{7 c}$ were obtained by reaction of compounds $\mathbf{6 a}, \mathbf{6 b}$, and $\mathbf{6 c}$, obtained by a

(29) Richman, J. E.; Atkins, T. J. J. Am. Chem. Soc. 1974, 96, 2268. standard tosylation procedure of the correspondent polyamines $\mathbf{5 a}, \mathbf{5 b}$, and $\mathbf{5} \mathbf{c}$, with 2 equiv of macrocycle $\mathbf{4}$. The final compounds $\mathbf{7 a}, \mathbf{7 b}$, and $\mathbf{7} \mathbf{c}$ must be purified from the crude products by flash chromatography. The cleavage reactions of the $p$-toluensulfonyl groups on the amine functions and of the methyl on the phenol were carried out under electron reduction conditions using lithium in liquid ammonia at $203 \mathrm{~K}$, obtaining, after the described process, the ligands $\mathbf{L 1}, \mathbf{L 2}$, and $\mathbf{L 3}$. The final compounds were further purified as hydrochloride salts by recrystallization from a methanol/ethanol mixture.

Description of the Structure. $\left\{\left[\mathrm{Cu}_{2} \mathrm{~L} 3\right]\left(\mathrm{ClO}_{4}\right)_{4}\right.$. $\left.3 \mathbf{H}_{2} \mathbf{O}\right\}_{2} \cdot \mathbf{H}_{2} \mathrm{O}(\mathbf{8})$. In the asymmetric unit of $\mathbf{8}$ two dinuclear complexes $\left[\mathrm{Cu}_{2} \mathbf{L} 3\right]^{4+}$ (Figure 1), hereafter indicated as $\mathbf{a}$ and $\mathbf{b}$, eight perchlorate anions, and seven water molecules are present. As illustrated below, all the species in the asymmetric unit are bound to each other through a net of strong hydrogen bonds, ${ }^{30}$ in this way forming a single independent unit. The two $\left[\mathrm{Cu}_{2} \mathbf{L} 3\right]\left(\mathrm{ClO}_{4}\right)_{4} \cdot 3 \mathrm{H}_{2} \mathrm{O}$ units are related by a non-crystallographic symmetry plane, ${ }^{31}$ which, as a consequence, also relates the two dinuclear metal complexes a and $\mathbf{b}$.

More precisely in each metal complex (Figure 1) both the copper cations are penta-coordinated by two nitrogen atoms of the ring (N2, N3 and N8, N9 for $\mathrm{Cu} 1$ and $\mathrm{Cu} 2$, respectively), two nitrogen atoms of the chain connecting the two rings (N4, N5 and N6, N7 for $\mathrm{Cu} 1$ and $\mathrm{Cu} 2$, respectively), and the phenolate oxygen atom $(\mathrm{O} 1$ and $\mathrm{O} 2$ for $\mathrm{Cu} 1$ and $\mathrm{Cu} 2$, respectively). Bond distances and angles about the metal cations are in keeping with those already reported in the literature for analogous metal complexes (see Table 2). The coordination polyhedron, in all the cases, can be described as a distorted square pyramid (the $\tau^{32}$ index is about 0.29 ) with $\mathrm{N} 3$ and $\mathrm{N} 8$ occupying the apical position about $\mathrm{Cu} 1$ and $\mathrm{Cu} 2$, respectively. The maximum deviation from the mean plane described by the four basal donors is 0.316(9) ( $\AA$ ) for N4 in complex $\mathbf{b}$. In all cases the copper ion is slightly shifted toward the apical donor (the largest displacement is 0.231(1) ( $\mathrm{A}$ ) for $\mathrm{Cu} 1$ in complex a, with the $\mathrm{Cu}-\mathrm{N}_{\text {apical }}$ bond almost perpendicular to the base of the square pyramid in both the complexes. Finally, the distances between the two copper atoms in the dinuclear complexes are 5.338(2) and 5.306(2) ^ for $\mathbf{a}$ and $\mathbf{b}$, respectively.

Ligand $\mathbf{L 3}$ is in a zwitterionic form, with the oxygen atoms of the phenol moieties deprotonated and the acidic hydrogen atoms bound to the uncoordinated nitrogen atoms N1 and N10 (see Figure 2) belonging to the macrocyclic units. The ligand shows an overall folded conformation with the planes defined by the two aromatic rings forming an angle of $6.0(2)$ and $3.5(3)^{\circ}$ for $\mathbf{a}$ and $\mathbf{b}$, respectively. The distances between the two centroids

(30) Jeffrey G. A. An introduction to hydrogen bonding; Oxford University Press: New York, 1997.

(31) The presence in the asymmetric unit of two $\left[\left(\mathrm{Cu}_{2} \mathrm{L3}\right)\left(\mathrm{ClO}_{4}\right)_{4} \cdot 3\left(\mathrm{H}_{2} \mathrm{O}\right)\right]$ units related by a noncrystallographic symmetry plane induces us to analyze several crystals of $\mathbf{8}$. The different sets of collected data were carefully checked to exclude mistakes in the crystal symmetry attribution and twinning phenomena. Finally, the presence of two $\left[\left(\mathrm{Cu}_{2} \mathbf{L} 3\right)\left(\mathrm{ClO}_{4}\right)_{4}\right.$. $3\left(\mathrm{H}_{2} \mathrm{O}\right)$ ] moieties in the asymmetric unit has been explained by considering that the bridging water molecule $(\mathrm{O} 4 \mathrm{w})$ creates a sort of dimer which behaves as an independent unit.

(32) Addison, A. W.; Rao, T. N.; Reedijk, J.; Van Rijn, J.; Vershcoor, G. C. J. Chem. Soc., Dalton Trans. 1984, 1349. 
Scheme 1. Synthesis of the Ligands

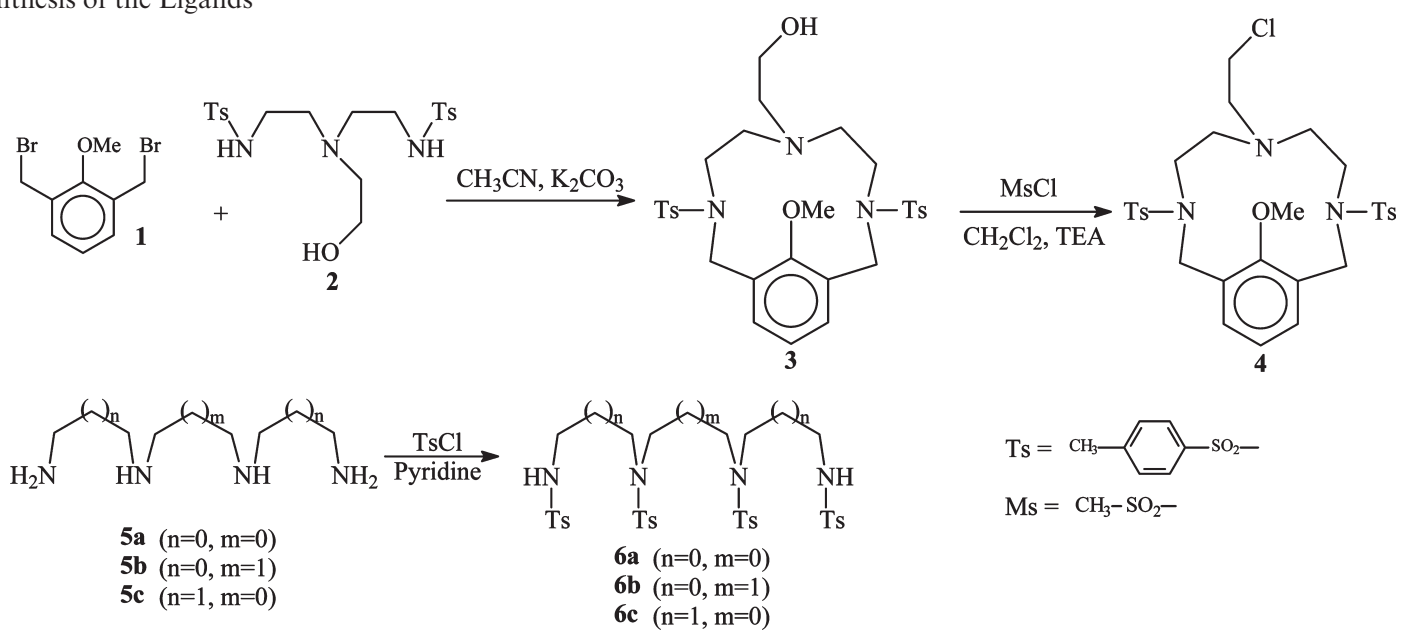

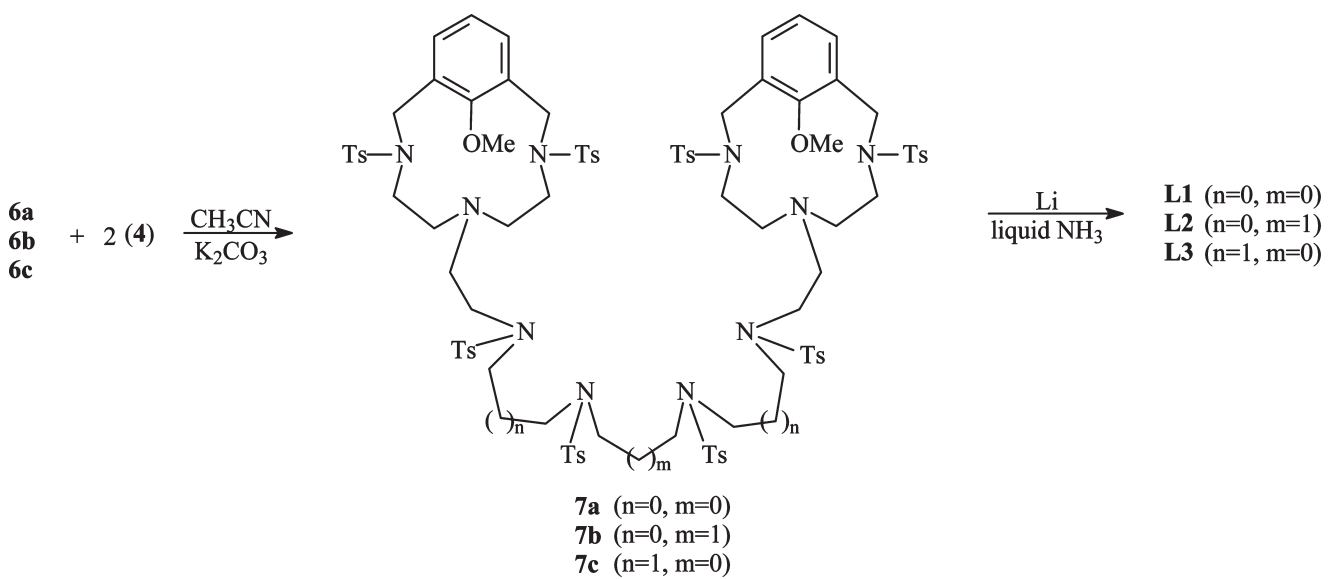

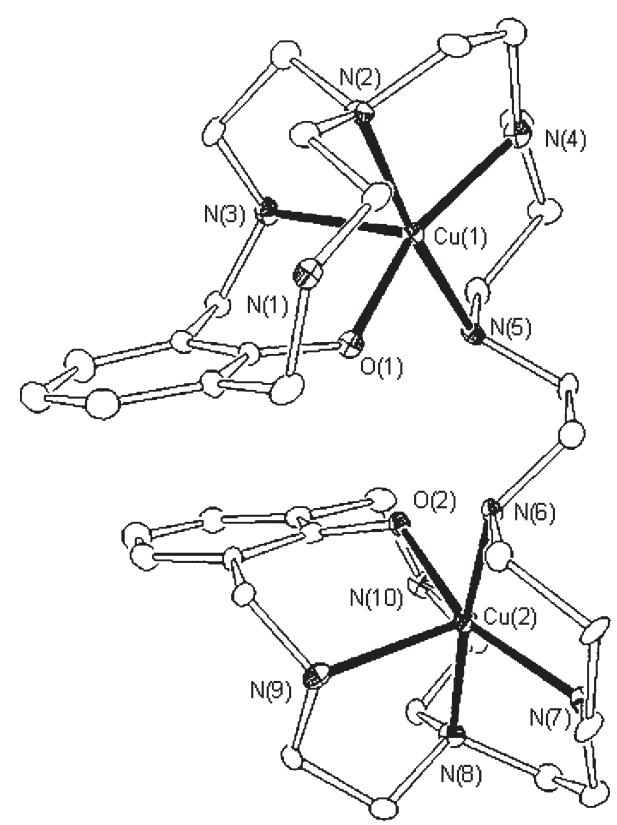

Figure 1. ORTEP 3 view of the complex cation $\left[\mathrm{Cu}_{2} \mathbf{L} 3\right]^{4+}$. Only one of the two dinuclear metal complexes of the asymmetric unit is represented. Hydrogen atoms are omitted for clarity. The ellipsoid probability is $20 \%$.

defined by the carbon atoms of the two rings are 4.875 and $5.053 \AA$ in a and $\mathbf{b}$, respectively.
Concerning the crystal packing, each $\left[\mathrm{Cu}_{2} \mathbf{L} 3\right]^{4+}$ species interacts with three water molecules, which in turn network four perchlorate anions, thus creating two $\left[\mathrm{Cu}_{2} \mathbf{L} 3\right]\left(\mathrm{ClO}_{4}\right)_{4} \cdot 3 \mathrm{H}_{2} \mathrm{O}$ units (see Table 3, Scheme 2, and Figure 2). These two moieties are further linked together by the seventh water molecule of crystallization (O4w) which, via hydrogen bonds, bridges the two dinuclear complexes.

More in detail (refer to Scheme 2 and Figure 2), each $\left[\mathrm{Cu}_{2} \mathbf{L} 3\right]\left(\mathrm{ClO}_{4}\right)_{4} \cdot 3 \mathrm{H}_{2} \mathrm{O}$ unit features the following pattern of H-bond interactions: the hydrogen atoms of the $\mathrm{N}$ (10) $\mathrm{H}_{2}$ grouping interact with two water molecules $(\mathrm{O} 1 \mathrm{w}$ and $\mathrm{O} 2 \mathrm{w}$ ). The latter molecules are held together also by a perchlorate anion (labeled as 3 in Figure 2). In addition the oxygen atom, $\mathrm{O} 1 \mathrm{w}$ interacts via hydrogen bonds with the two perchlorate anions 1 and 4 (see Figure 2) too. Finally the third water molecule of the $\left[\mathrm{Cu}_{2} \mathbf{L} 3\right]\left(\mathrm{ClO}_{4}\right)_{4}$. $3 \mathrm{H}_{2} \mathrm{O}$ unit, namely, $\mathrm{O} 3 \mathrm{w}$, interacts with one hydrogen atom of the $\mathrm{N}(1) \mathrm{H}_{2}$ grouping. This water molecule is then linked to a perchlorate anion (2 in Figure 2).

The interaction of the two $\left[\mathrm{Cu}_{2} \mathbf{L} 3\right]\left(\mathrm{ClO}_{4}\right)_{4} \cdot 3 \mathrm{H}_{2} \mathrm{O}$ moieties with the same water molecule $(\mathrm{O} 4 \mathrm{w})$ gives rise to the whole independent crystallographic unit discussed above. ${ }^{31}$

Finally, the H-bond interactions further extend the asymmetric unit (see Table 3 ) so that a net of hydrogen interactions is present all through the crystal.

Solution Studies. Basicity. Table 4 summarizes the basicity constants of $\mathbf{L 1}, \mathbf{L} 2$, and $\mathbf{L 3}$ potentiometrically 


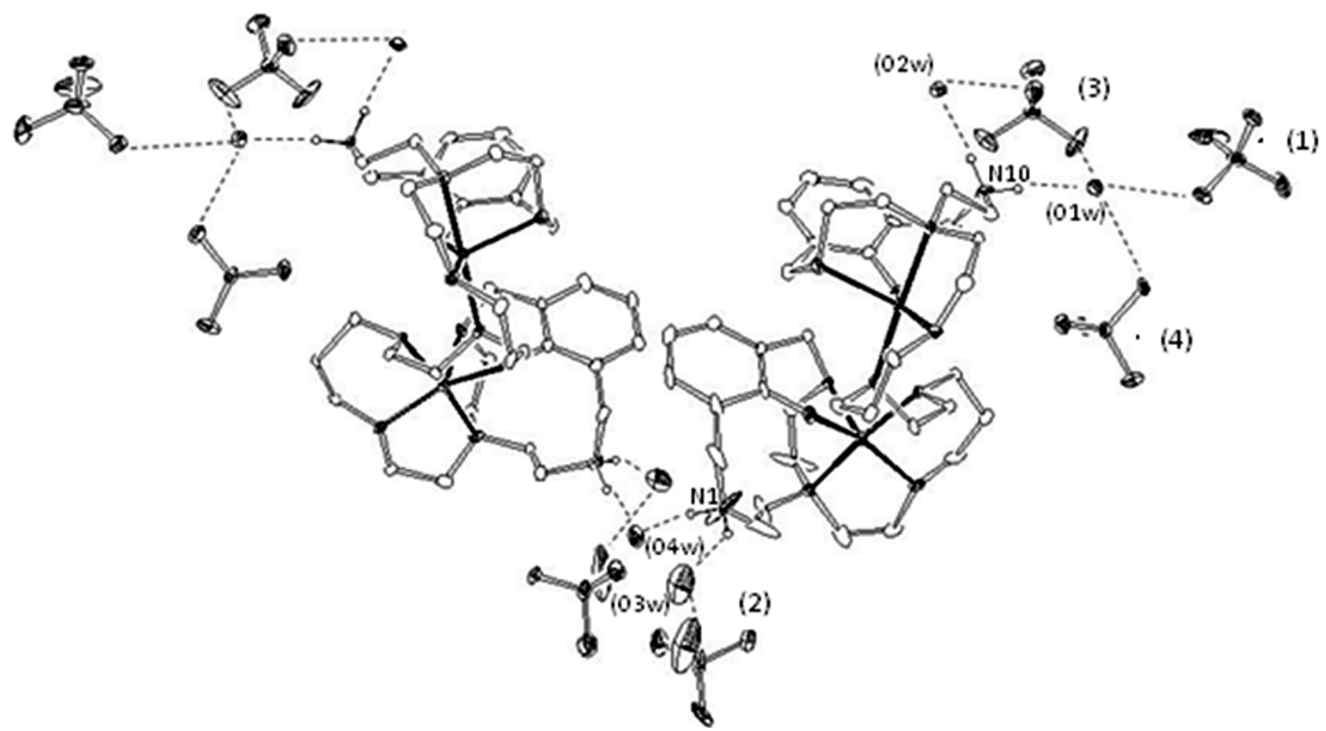

Figure 2. ORTEP 3 view of the asymmetric unit of $\mathbf{8}$ showing the H-bond network involving the metal complexes, the counterions, and the crystallization water molecules.

Table 3. Intermolecular Hydrogen Bonds $(\AA)$ in $\mathbf{8}$

\begin{tabular}{ll}
\hline $\mathbf{a}^{a}$ & $\mathbf{b}$ \\
\hline
\end{tabular}

$\mathrm{H}$-bond interactions inside each $\left[\mathrm{Cu}_{2} \mathbf{L} 3\right]\left(\mathrm{ClO}_{4}\right]_{4} \cdot 3\left(\mathrm{H}_{2} \mathrm{O}\right)$ unit

$\begin{array}{lll}\mathrm{N} 10 \cdots \mathrm{O} 1 \mathrm{w} & 2.858(8)^{b} & 2.837(9) \\ \mathrm{N} 10 \cdots \mathrm{O} 2 \mathrm{w} & 2.830(8) & 2.806(9) \\ \mathrm{N} 1 \cdots \mathrm{O} 3 \mathrm{w} & 2.81(1) & 2.70(2) \\ \mathrm{O} 1 \mathrm{w} \cdots \mathrm{O} 14 & 2.829(9) & 2.817(9) \\ \mathrm{O} 1 \mathrm{w} \cdots \mathrm{O} 32 & 2.90(1) & 2.893(9) \\ \mathrm{O} 1 \mathrm{w} \cdots \mathrm{O} 41 & 3.057(8) & 2.963(8) \\ \mathrm{O} 2 \mathrm{w} \cdots \mathrm{O} 31 & 3.017(9) & 2.995(8) \\ \mathrm{O} 3 \mathrm{w} \cdots \mathrm{O} 24 & 2.75(1) & 3.212(2)\end{array}$

H-bond interaction connecting the two $\left[\mathrm{Cu}_{2} \mathbf{L} 3\right]\left(\mathrm{ClO}_{4}\right]_{4} \cdot 3\left(\mathrm{H}_{2} \mathrm{O}\right)$ units
$\mathrm{N} 1 \cdots \mathrm{O} 4 \mathrm{w}$
$2.82(1)$
$3.42(2)$

$\mathrm{H}$-bond interactions extending beyond the asymmetric unit

\begin{tabular}{|c|c|c|}
\hline $\mathrm{N} 3 \cdots \mathrm{O} 14^{c}$ & $3.073(9)$ & $3.033(8)$ \\
\hline $\mathrm{N} 7 \cdots \mathrm{O} 12^{d}$ & $3.073(9)$ & $3.036(9)$ \\
\hline $\mathrm{O} 3 \mathrm{wa} \cdot \mathrm{O} 3 \mathrm{wb}^{e}$ & & \\
\hline $\mathrm{O} 2 \mathrm{wb} \cdots \mathrm{O} 42 \mathrm{a}^{f}$ & & \\
\hline $\mathrm{O} 2 \mathrm{wa} \cdots \mathrm{O} 43 \mathrm{~b}^{g}$ & & \\
\hline $\mathrm{O} 2 \mathrm{wa} \cdots \mathrm{O} 1 \mathrm{wb}^{g}$ & & \\
\hline $\mathrm{O} 1 \mathrm{wa} \cdot \mathrm{O} 2 \mathrm{wb}^{g}$ & & \\
\hline $\mathrm{O} 4 \mathrm{w} \cdot \mathrm{O} 44 \mathrm{a}^{h}$ & & \\
\hline $\mathrm{O} 3$ wa $\cdots \mathrm{O}_{44 \mathrm{~b}^{i}}$ & & \\
\hline
\end{tabular}

${ }^{a}$ The $\mathbf{a}$ and $\mathbf{b}$ labels refer to the two independent $\left[\mathrm{Cu}_{2} \mathbf{L} 3\right]-$ $\left(\mathrm{ClO}_{4}\right]_{4} \cdot 3\left(\mathrm{H}_{2} \mathrm{O}\right)$ moieties found in the asymmetric unit. ${ }^{b}$ Values in parentheses are the standard deviations on the last significant figure. ${ }^{c} x+1 / 2,-y+3 / 2,-z+1$ (a), $x+1 / 2,-y+3 / 2,-z$ (b). ${ }^{d} x-1 / 2,-y+$ $3 / 2,-z+1$ (a), $x-1 / 2,-y+3 / 2,-z(\mathrm{~b}) .{ }^{e} x+1, y, z{ }^{f}-x+1 / 2,-y+2$, $z-1 / 2 .{ }^{g}-x+1 / 2,-y+2, z+1 / 2 .{ }^{h}$ Same as $d(\mathrm{a})=x-1 / 2,-y+3 / 2$, $-z+1 .{ }^{i}$ Same as $c(\mathrm{~b})=x+1 / 2,-y+3 / 2,-z$.

determined in $0.15 \mathrm{~mol} \mathrm{dm}^{-3} \mathrm{NMe}_{4} \mathrm{Cl}$ aqueous solution at $298.1 \mathrm{~K}$. All the ligands contain 10 amine and two phenol functions, thus potentially behaving as a decaprotic base and diprotic acid. Starting from the L neutral species, all the possible protonation species cannot be reached in the examined $2-12 \mathrm{pH}$ range; potentiometric data show that neutral L species of L1-L3 undergoes a total of eight protonation steps, while deprotonation of the free neutral L is not detected. L1 shows three rather high protonation constants $\left(\log K_{1-3}=10.06,8.98\right.$, and
Scheme 2. Schematic Drawing of the H-Bond Interactions Involving the Species within the Asymmetric Unit of 8

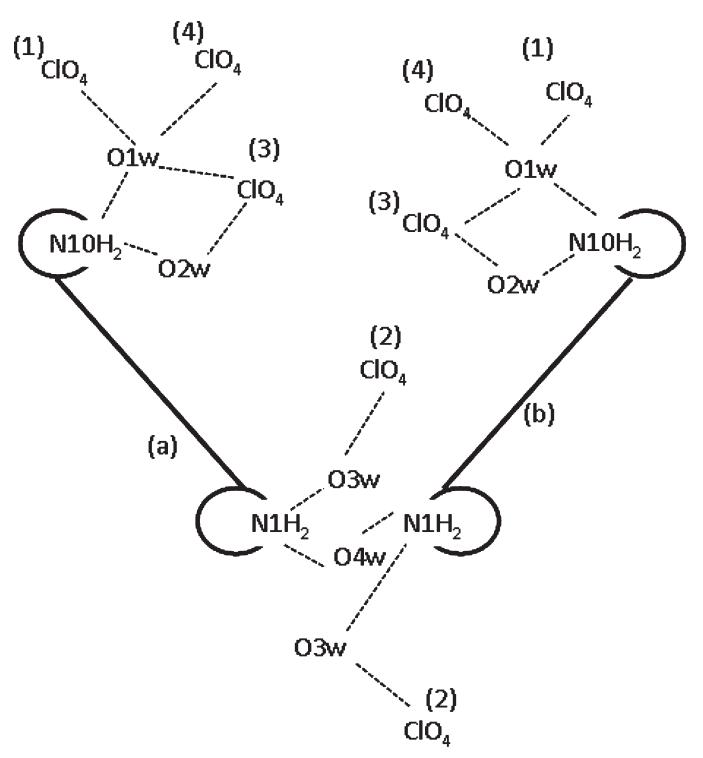

8.08) decreasing by about one logarithmic unit for each step; on the contrary, the fourth step $\left(\log K_{4}=6.31\right)$ is 1.7 logarithmic units less than the third one. The following two steps show similar constants $\left(\log K_{5-6}=4.96\right.$ and 4.33 ), and the last two steps show low constant values (log $K_{7-8}=3.02$ and 2.61). L2 and L3 show similar basicity, although they are a bit more basic than $\mathbf{L} \mathbf{1}$ in all protonation steps (Table 4). This behavior can be easily ascribed to the presence of the longer propyl chain in L2 and L3 rather than all ethyl chains in $\mathbf{L} \mathbf{1}$ which better allows the minimization of the electrostatic repulsions in the protonated species.

All three ligands contain two L4 amino-phenolic macrocyclic subunits spaced by linear tetraamines. As mentioned above, the fully deprotonated $\mathrm{H}_{-2} \mathrm{~L}^{2-}$ species was not observed for all the ligands; considering that the free neutral $\mathbf{L} \mathbf{4}$ species loses the acidic proton to form the $\mathrm{H}_{-1} \mathbf{L 4}^{-}$species and that the first proton addition to this 
Table 4. Protonation Constants $(\log K)$ of Ligands $\mathbf{L} 1, \mathbf{L} 2$, and $\mathbf{L} 3$ Potentiometrically Determined in $0.15 \mathrm{~mol} \mathrm{dm}^{-3} \mathrm{NMe}_{4} \mathrm{Cl}$ Aqueous Solution at $298.1 \mathrm{~K}$

\begin{tabular}{lccr}
\hline & \multicolumn{3}{c}{$\log K$} \\
\cline { 2 - 4 } \multicolumn{1}{c}{ reaction } & \multicolumn{1}{c}{$\mathbf{L}$} & $\mathbf{L} 2$ & \multicolumn{1}{c}{$\mathbf{L 3}$} \\
\hline $\mathbf{L}+\mathrm{H}^{+}=\mathrm{HL}^{+}$ & $10.06(1)^{a}$ & $10.56(1)$ & $10.57(1)$ \\
$\mathrm{HL}^{+}+\mathrm{H}^{+}=\mathrm{H}_{2} \mathbf{L}^{2+}$ & $8.98(1)$ & $9.28(1)$ & $9.51(1)$ \\
$\mathrm{H}_{2} \mathbf{L}^{2+}+\mathrm{H}^{+}=\mathrm{H}_{3} \mathbf{L}^{3+}$ & $8.08(2)$ & $8.49(1)$ & $8.57(1)$ \\
$\mathrm{H}_{3} \mathbf{L}^{3+}+\mathrm{H}^{+}=\mathrm{H}_{4} \mathbf{L}^{4+}$ & $6.31(2)$ & $7.24(2)$ & $7.61(2)$ \\
$\mathrm{H}_{4} \mathbf{L}^{4+}+\mathrm{H}^{+}=\mathrm{H}_{5} \mathbf{L}^{5+}$ & $4.96(2)$ & $5.54(2)$ & $5.90(2)$ \\
$\mathrm{H}_{5} \mathbf{L}^{5+}+\mathrm{H}^{+}=\mathrm{H}_{6} \mathbf{L}^{6+}$ & $4.33(3)$ & $4.98(2)$ & $4.87(2)$ \\
$\mathrm{H}_{6} \mathbf{L}^{6+}+\mathrm{H}^{+}=\mathrm{H}_{7} \mathbf{L}^{7+}$ & $3.02(3)$ & $4.01(3)$ & $4.39(3)$ \\
$\mathrm{H}_{7} \mathbf{L}^{7+}+\mathrm{H}^{+}=\mathrm{H}_{8} \mathbf{L}^{8+}$ & $2.61(3)$ & $2.71(3)$ & $2.44(3)$ \\
\multicolumn{2}{c}{ Values in parentheses } & are the standard deviations on the last \\
significant figure. & & &
\end{tabular}

latter almost reaches the limit of the method in these experimental conditions $\left(\log K_{1}=11.66\right),{ }^{10 \mathrm{~b}}$ the connection of two $\mathbf{L} 4$ units to form $\mathbf{L} \mathbf{-}-\mathbf{L} 3$ ligands probably increases the basicity of the $\mathbf{L} \mathbf{4}$ subunits making the full deprotonation of the two macrocycles thus that of the ligands undetectable. It is noteworthy that the neutral $\mathbf{L 4}$ species is in the zwitterionic form with the amine group in the benzyl position protonated and the phenol deprotonated. ${ }^{10 \mathrm{~b}}$

UV-vis absorption electronic spectra were carried out in the same experimental conditions at different $\mathrm{pH}$ values to understand the role of the phenolic functions in the acid-base behavior of the ligands. The aza-phenolic macrocycles exhibit different spectral features when the phenol function is deprotonated or in the neutral form. In the latter, it shows a $\lambda_{\max }$ at approximately $277 \mathrm{~nm}$, while it shows two bands with $\lambda_{\max }$ at approximately 242 and $298 \mathrm{~nm}$ in the deprotonated phenolate form..$^{9 \mathrm{c}, \mathrm{d}, 10 \mathrm{~b}}$

Figure 3 reports the trend of the absorbance values at $\lambda=298 \mathrm{~nm}$, typical of the anionic phenolate group, together with the distribution diagram of the protonated species of $\mathbf{L 1}-\mathbf{L} \mathbf{3}$ as a function of $\mathrm{pH}$. Monitoring the spectra of $\mathbf{L 1}$ from the basic to the acid $\mathrm{pH}$ region (i.e., from phenolate to phenol form), it results that the absorption at $\lambda_{\max }=298 \mathrm{~nm}$ is rather constant from $\mathrm{pH}=$ 12 to $\mathrm{pH}=6.5$, and then it decreases up to $\mathrm{pH}=3$ and remains constant below this value. Merging these data with the distribution diagram of the species, it is possible to point out that the phenolate absorption starts to drop when the $\mathrm{H}_{5} \mathbf{L} \mathbf{1}^{5+}$ species appears in solution, and it reaches the baseline when the $\mathrm{H}_{6} \mathbf{L 1}^{6+}$ species is fully formed (Figure 3a). On the basis of these considerations, a scheme for the form of the phenol groups in the species of $\mathbf{L} \mathbf{1}$ can be outlined: in the species from $\mathbf{L} \mathbf{1}$ to $\mathrm{H}_{4} \mathbf{L 1}^{4+}$ they are both present as phenolates; on the contrary, in the species from $\mathrm{H}_{6} \mathbf{L} \mathbf{1}^{6+}$ to $\mathrm{H}_{8} \mathbf{L} \mathbf{1}^{8+}$ they are both present as neutral phenols; finally, in the $\mathrm{H}_{5} \mathbf{L} \mathbf{1}^{5+}$ species they are present one as a phenol and one as a phenolate group. Similar remarks can be made for $\mathbf{L} 2$ and $\mathbf{L} 3$ analyzing the Figure 3b,c. In these cases, the two groups resulted as phenolate in all the species from $\mathbf{L}$ to $\mathrm{H}_{5} \mathbf{L}^{5+}$, as phenol in the species $\mathrm{H}_{7} \mathbf{L}^{7+}$ and $\mathrm{H}_{8} \mathbf{L}^{8+}$, while in the species $\mathrm{H}_{6} \mathbf{L}^{6+}$ only one of the two phenol groups is in the anionic form.

In other words, the main difference between $\mathbf{L} 1$ and the other two ligands is that the number of amine functions
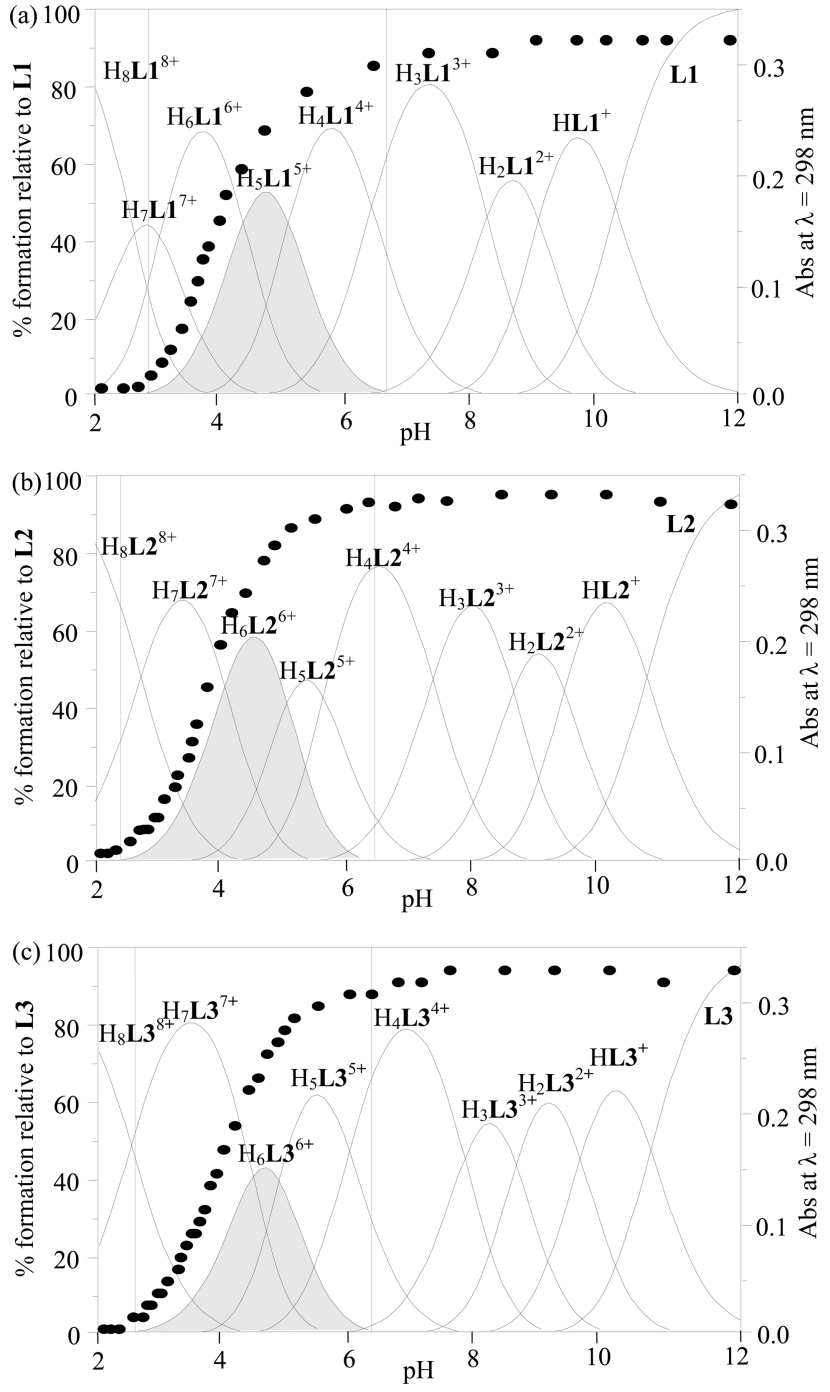

Figure 3. Distribution curves of the species (-) and absorption values at $\lambda=298 \mathrm{~nm}(\mathbf{O})$ of $\mathbf{L 1}$ (a), $\mathbf{L 2}$ (b), and $\mathbf{L 3}$ (c) as a function of $\mathrm{pH}$ in aqueous solution at $298.1 \mathrm{~K}, I=0.15 \mathrm{M} \mathrm{NMe}_{4} \mathrm{Cl}$; $[\mathrm{L}]=4 \times 10^{-5} \mathrm{M}$. The areas filled in gray indicate the species where only one phenol moiety is deprotonated.

that can be protonated before the phenolate are six for $\mathbf{L 1}$, while they are seven for $\mathbf{L} \mathbf{2}$ and $\mathbf{L} 3$.

In fact, it is noteworthy that all neutral $\mathbf{L 1}-\mathbf{L} 3$ species are amphionic and that, being the two aromatic groups present as phenolate anions, they must have two protonated ammonium groups (Figure 4a); this proton disposition is suggested by similar results found for the neutral species of $\mathbf{L} \mathbf{4}$.

Concluding, L1, L2, and $\mathbf{L 3}$ show similar acid-base behavior, although as previously discussed, the presence of the shorter amino-aliphatic spacer chain decreases the basicity of $\mathbf{L} \mathbf{1}$ with respect to $\mathbf{L} \mathbf{2}$ and $\mathbf{L} 3$ in all protonation steps, thus involving the phenolate groups of $\mathbf{L} \mathbf{1}$ in protonation at higher $\mathrm{pH}$ values than the others as well as in different protonated species.

Coordination of $\mathbf{C u}(\mathbf{I I})$. Table 5 summarizes the stability constants of $\mathbf{L} \mathbf{1}-\mathbf{L} 3$ with the $\mathrm{Cu}(\mathrm{II})$ ion, potentiometrically determined in $0.15 \mathrm{~mol} \mathrm{dm}^{-3} \mathrm{NMe}_{4} \mathrm{Cl}$ aqueous solution at $298.1 \mathrm{~K}$. All ligands form mono-, di-, and trinuclear complexes.

Mononuclear Complexes. Amphionic species L1-L3 add a $\mathrm{Cu}(\mathrm{II})$ ion giving rise to mononuclear $[\mathrm{CuL}]^{2+}$ 


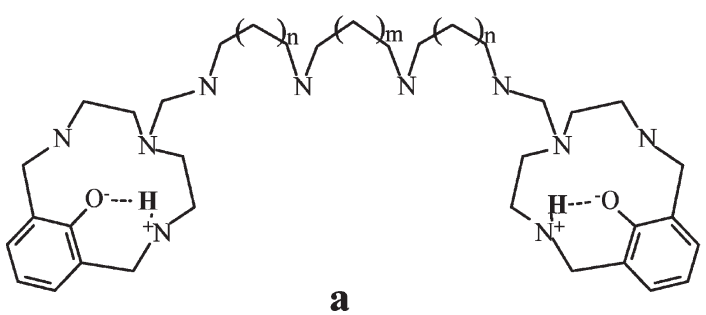

$\mathbf{a}$

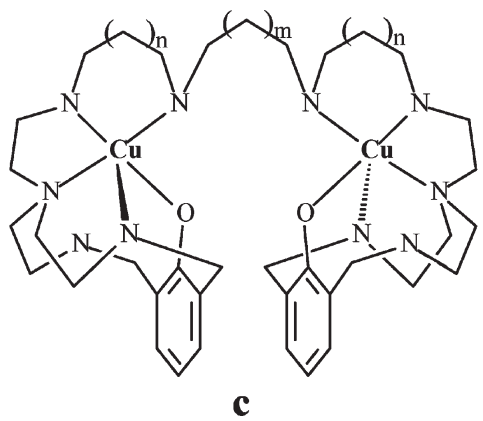

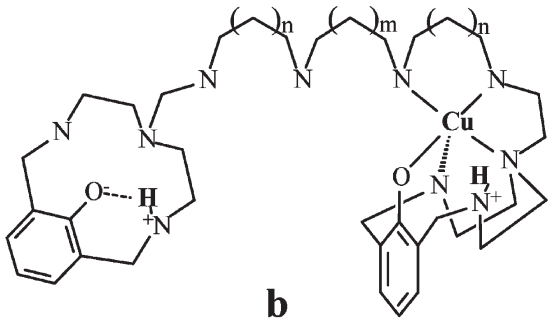

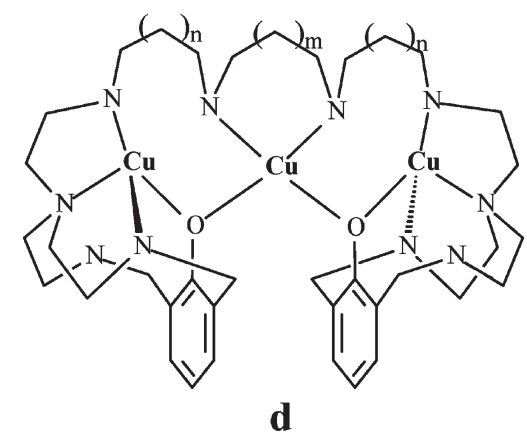

Figure 4. Proposed models for neutral $\mathrm{L}$ (a), mononuclear $[\mathrm{CuL}]^{2+}$ (b), dinuclear $\left[\mathrm{Cu}_{2} \mathrm{H}_{-2} \mathrm{~L}\right]^{2+}$ (c), and trinuclear $\left[\mathrm{Cu}_{3} \mathrm{H}_{-2} \mathbf{L}\right]^{4+}(\mathrm{d}) \operatorname{species}(n=0, m=0$, $\mathrm{L}=\mathbf{L} 1 ; n=0, m=1, \mathrm{~L}=\mathbf{L} 2 ; n=1, m=0, \mathbf{L}=\mathbf{L} 3$.

Table 5. Equilibrium Constants $(\log K)$ for the $\mathrm{Cu}(\mathrm{II})$ Metal Complexes Equilibria of L1, L2, and $\mathbf{L 3}$ Potentiometrically Determined in $0.15 \mathrm{~mol} \mathrm{dm}^{-3}$ $\mathrm{NMe}_{4} \mathrm{Cl}$ Aqueous Solution at $298.1 \mathrm{~K}$

\begin{tabular}{|c|c|c|c|}
\hline \multirow[b]{2}{*}{ reaction } & \multicolumn{3}{|c|}{$\log K$} \\
\hline & L1 & L2 & L3 \\
\hline $\mathbf{L}+\mathrm{Cu}^{2+}=\mathrm{Cu}^{2+}$ & $20.03(1)^{a}$ & $21.18(1)$ & $19.26(1)$ \\
\hline $\mathrm{CuL}^{2+}=\mathrm{CuH}_{-1} \mathbf{L}^{+}+\mathrm{H}^{+}$ & $-9.30(3)$ & $-9.51(2)$ & $-9.53(3)$ \\
\hline $\mathrm{CuL}^{2+}+\mathrm{H}^{+}=\mathrm{CuHL}^{3+}$ & $8.22(2)$ & $8.95(2)$ & $8.53(1)$ \\
\hline $\mathrm{CuHL}^{3+}+\mathrm{H}^{+}=\mathrm{CuH}_{2} \mathbf{L}^{4+}$ & $5.76(1)$ & $6.86(3)$ & $7.00(2)$ \\
\hline $\mathrm{CuH}_{2} \mathbf{L}^{4+}+\mathrm{H}^{+}=\mathrm{CuH}_{3} \mathbf{L}^{5+}$ & $4.12(1)$ & $4.80(2)$ & $5.06(2)$ \\
\hline $\mathrm{CuH}_{3} \mathbf{L}^{5+}+\mathrm{H}^{+}=\mathrm{CuH}_{4} \mathbf{L}^{6+}$ & $2.31(2)$ & $2.65(2)$ & $4.37(1)$ \\
\hline $\mathrm{CuH}_{4} \mathbf{L}^{6+}+\mathrm{H}^{+}=\mathrm{CuH}_{5} \mathbf{L}^{7+}$ & & & $3.42(3)$ \\
\hline $\mathrm{CuH}_{-1} \mathbf{L}^{+}+\mathrm{Cu}^{2+}=\mathrm{Cu}_{2} \mathrm{H}_{-1} \mathbf{L}^{3+}$ & $14.82(2)$ & $15.25(2)$ & $15.34(2)$ \\
\hline $\mathrm{CuL}^{2+}+\mathrm{Cu}^{2+}=\mathrm{Cu}_{2} \mathbf{L}^{4+}$ & $11.53(2)$ & $11.99(3)$ & $12.10(2)$ \\
\hline $\mathrm{Cu}_{2} \mathrm{H}_{-1} \mathbf{L}^{3+}=\mathrm{Cu}_{2} \mathrm{H}_{-2} \mathbf{L}^{2+}+\mathrm{H}^{+}$ & $-9.64(3)$ & $-8.93(3)$ & $-8.67(3)$ \\
\hline $\begin{array}{l}\mathrm{Cu}_{2} \mathrm{H}_{-2} \mathbf{L}^{2+}+\mathrm{OH}^{-} \\
\quad=\mathrm{Cu}_{2} \mathrm{H}_{-2} \mathbf{L}(\mathrm{OH})^{+}\end{array}$ & $4.66(3)$ & 4.44(3) & $4.58(3)$ \\
\hline $\begin{array}{l}\mathrm{Cu}_{2} \mathrm{H}_{-2} \mathbf{L}(\mathrm{OH})^{+}+\mathrm{OH}^{-} \\
\quad=\mathrm{Cu}_{2} \mathrm{H}_{-2} \mathbf{L}(\mathrm{OH})_{2}\end{array}$ & & $3.89(3)$ & $3.52(3)$ \\
\hline $\mathrm{Cu}_{2} \mathbf{L}^{4+}+\mathrm{Cu}^{2+}=\mathrm{Cu}_{3} \mathbf{L}^{6+}$ & $3.89(2)$ & $5.51(2)$ & $4.39(2)$ \\
\hline $\mathrm{Cu}_{2} \mathrm{H}_{-2} \mathbf{L}^{2+}+\mathrm{Cu}^{2+}=\mathrm{Cu}_{3} \mathrm{H}_{-2} \mathbf{L}^{4+}$ & $8.19(2)$ & $9.64(2)$ & $8.82(2)$ \\
\hline $\mathrm{Cu}_{3} \mathrm{H}_{-2} \mathbf{L}^{4+}+\mathrm{H}^{+}=\mathrm{Cu}_{3} \mathrm{H}_{-1} \mathbf{L}^{5+}$ & $6.96(2)$ & $6.88(2)$ & $5.43(2)$ \\
\hline $\mathrm{Cu}_{3} \mathrm{H}_{-1} \mathbf{L}^{5+}+\mathrm{H}^{+}=\mathrm{Cu}_{3} \mathbf{L}^{6+}$ & $4.39(2)$ & $4.17(3)$ & $5.09(2)$ \\
\hline $\begin{array}{l}\mathrm{Cu}_{3} \mathrm{H}_{-2} \mathbf{L}^{4+}+\mathrm{OH}^{-} \\
\quad=\mathrm{Cu}_{3} \mathrm{H}_{-2} \mathbf{L}(\mathrm{OH})^{3+}\end{array}$ & $6.01(2)$ & $6.00(3)$ & $6.64(2)$ \\
\hline $\begin{array}{l}\mathrm{Cu}_{3} \mathrm{H}_{-2} \mathrm{LOH}^{3+}+\mathrm{OH}^{-} \\
\quad=\mathrm{Cu}_{3} \mathrm{H}_{-2} \mathbf{L}(\mathrm{OH})_{2}^{2+}\end{array}$ & $3.32(2)$ & & $5.45(2)$ \\
\hline
\end{tabular}

species ( $\mathbf{L}=\mathbf{L 1}, \mathbf{L 2}$, and $\mathbf{L 3}$ ) with formation constant values of approximately $20 \operatorname{logarithmic}$ units $(\log K)$. The distribution diagrams of the species as a function of $\mathrm{pH}$ (see Supporting Information Figure S1) show that the mononuclear prevails over the polynuclear species for the $\mathrm{L} / \mathrm{Cu}(\mathrm{II})$ molar ratio of $1: 1$, although all mono- and polynuclear complexes are present in solution. The $[\mathrm{CuL}]^{2+}$ species can add four or five protons reaching the polyprotonated $\left[\mathrm{CuH}_{4} \mathbf{L 1}\right]^{6+},\left[\mathrm{CuH}_{4} \mathbf{L} 2\right]^{6+}$, and $\left[\mathrm{CuH}_{5} \mathbf{L} \mathbf{3}\right]^{7+}$ species, respectively, or it can lose one proton forming the $\left[\mathrm{CuH}_{-1} \mathrm{~L}\right]^{+}$species; further deprotonation processes were not observed. The high values for the formation of $[\mathrm{CuL}]^{2+}$ species suggest the involvement of several binding groups in stabilizing the $\mathrm{Cu}(\mathrm{II})$.

Examining the formation constant of the $\left[\mathrm{CuH}_{-1} \mathrm{~L}\right]^{+}$ species as a deprotonation reaction $[\mathrm{CuL}]^{2+}=\left[\mathrm{CuH}_{-1}\right.$ $\mathrm{L}^{+}+\mathrm{H}^{+}(\log K=-9.30,-9.51$, and -9.53 for $\mathbf{L 1}, \mathbf{L 2}$, and $\mathbf{L} 3$ respectively), the values found are in agreement with the deprotonation of a secondary ammonium group. ${ }^{33}$ This suggests, taking into account the distribution of the acidic protons in the neutral L species discussed above, that this process involves one of the two remaining acidic protons located in one of the $\mathbf{L} \mathbf{4}$ subunits; in addition, it also implies that this macrocyclic subunit is the part involved in the stabilization of the first $\mathrm{Cu}(\mathrm{II})$ as schematically reported for the $[\mathrm{CuL}]^{2+}$ species in Figure 4b. This hypothesis is supported (i) by the absence of a further deprotonation process of the $\left[\mathrm{CuH}_{-1} \mathrm{~L}\right]^{+}$species (no formation of hydroxylated species) indicating saturation of the coordination requirement of the metal ion; (ii) by the studies carried out on $\mathbf{L 4}$ showing that, due to steric hindrance, it behaves as a tridentate ligand with the involvement of only two secondary amine and the phenolate functions in the stabilization of the $\mathrm{Cu}$ (II) ion; ${ }^{10 \mathrm{a}}$ and (iii) by the high number of the protonated $\mathrm{Cu}(\mathrm{II})$ species in agreement with the fact that a large part of the molecule is not involved in the coordination. This statement is also supported by the observation of the crystal structure reported in Figure 1; in fact, half of the molecule is sufficient to fully stabilize one $\mathrm{Cu}(\mathrm{II})$, thus suggesting that in the $[\mathrm{CuL}]^{4+}$ and $\left[\mathrm{CuH}_{-1} \mathrm{~L}\right]^{3+}$ mononuclear species, the $\mathrm{Cu}(\mathrm{II})$ is coordinated in a similar way to one of the metal cations of the structure in Figure 1. In other words, the coordination of

(33) Bencini, A.; Bianchi, A.; Garcia-España, E.; Micheloni, M.; Ramirez, J. A. Coord. Chem. Rev. 1999, 188, 97. 
$\mathrm{Cu}$ (II) involves one of the $\mathbf{L} \mathbf{4}$ subunits with the phenolate oxygen and two nitrogen atoms and a part of the polyamine chain, while the other part of the molecule can undergo protonation (see Figure $4 b$ ).

Dinuclear Complexes. The distribution diagrams of the species as a function of $\mathrm{pH}$ (see Supporting Information, Figure S2) show that the dinuclear species prevail over the others in a large range of $\mathrm{pH}(6<\mathrm{pH}<12)$ for $\mathrm{L}$ to $\mathrm{Cu}$ (II) molar ratio of 1 to 2 . All the mononuclear $\left[\mathrm{CuH}_{-1} \mathrm{~L}\right]^{+}$species are able to add another $\mathrm{Cu}(\mathrm{II})$ giving rise to dinuclear $\left[\mathrm{Cu}_{2} \mathrm{H}_{-1} \mathrm{~L}\right]^{3+}$ complexes with rather high constants $(\log K=14.82,15.25$, and 15.34 for $\mathbf{L 1}, \mathbf{L 2}$, and $\mathbf{L 3}$, respectively, see Table 5). The addition of the second $\mathrm{Cu}$ (II) to the $[\mathrm{CuL}]^{2+}$ species shows similar values to the former addition, thus suggesting that the coordination of the second ion does not deeply affect the first $\mathrm{Cu}(\mathrm{II})$ ion environment (Table 5). This supports the idea that the ligands bind the two $\mathrm{Cu}(\mathrm{II})$ independently; that is, each metal ion is stabilized by one of the macrocyclic subunits and by some nitrogen atoms of the polyamine chain closer to the macrocycle (see Figure 4c). Moreover, the $\left[\mathrm{Cu}_{2} \mathrm{H}_{-1} \mathrm{~L}\right]^{3+}$ species easily undergoes a deprotonation process to generate the $\left[\mathrm{Cu}_{2} \mathrm{H}_{-2} \mathrm{~L}\right]^{2+}$ species, and the values of the constants related to this process are similar to those previously discussed for the deprotonation of the mononuclear $[\mathrm{CuL}]^{2+}$ species (Table 5); thus, also in this case, it is possible to suppose that the $\left[\mathrm{Cu}_{2} \mathrm{H}_{-1} \mathrm{~L}\right]^{3+}$ complex cation loses the last acidic proton located on the macrocyclic subunit as previously suggested for the $[\mathrm{CuL}]^{2+}$ species. In other words, a coordination environment for both $\mathrm{Cu}(\mathrm{II})$ ions in solution, similar to that shown in the crystal structure of the $\left[\mathrm{Cu}_{2} \mathbf{L} 3\right]^{4+}$ cation (see Figure 1), can be supposed not only for the $\left[\mathrm{Cu}_{2} \mathrm{~L}\right]^{4+}$ but also for the $\left[\mathrm{Cu}_{2} \mathrm{H}_{-1} \mathrm{~L}\right]^{3+}$ and $\left[\mathrm{Cu}_{2} \mathrm{H}_{-2} \mathrm{~L}\right]^{2+}$ species (Figure 4c). All the dinuclear complexes are able to form hydroxylated species, and the constant values related to the addition of $\mathrm{OH}^{-}$to the $\left[\mathrm{Cu}_{2} \mathrm{H}_{-2} \mathrm{~L}\right]^{2+}$ species are reported in Table 5. The values of this process are in the range of the addition of $\mathrm{OH}^{-}$to a $\mathrm{Cu}$ (II) complex (constant values ranging from 4.66 to 3.52 logarithmic units (Table 5)), thus suggesting that the $\mathrm{Cu}$ (II) ions are located too far away to be able to bind the hydroxide in a bridge disposition.

Trinuclear Complexes. As a result of the high number of donor atoms and to the flexibility given by the presence of the linear polyamine moiety, all ligands $\mathbf{L} \mathbf{1}-\mathbf{L} \mathbf{3}$ are able to form stable trinuclear complexes; these species are predominant in a large range of $\mathrm{pH}$ for the $\mathrm{L}$ to $\mathrm{Cu}$ (II) molar ratio of 1 to 3; the distribution diagrams of the species as a function of $\mathrm{pH}$ for all the ligands in this molar ratio are reported in Figure 5.

The addition constants of the third $\mathrm{Cu}(\mathrm{II})$ ion to the dinuclear $\left[\mathrm{Cu}_{2} \mathrm{H}_{-2} \mathrm{~L}\right]^{2+}$ species are $\log K=8.19,9.64$, and 8.82 for $\mathbf{L}=\mathbf{L} 1, \mathbf{L 2}$, and $\mathbf{L 3}$, respectively. These values are rather high considering they are related to the addition of a cation to a positively charged species, thus suggesting the involvement of several donor groups in stabilizing the third $\mathrm{Cu}(\mathrm{II})$. However, the three $\mathrm{Cu}(\mathrm{II})$ ions do not involve all the donor groups of the ligands in the coordination; in fact the $\left[\mathrm{Cu}_{3} \mathrm{H}_{-2} \mathrm{~L}\right]^{4+}$ trinuclear species can undergo two protonation steps yielding the $\left[\mathrm{Cu}_{3} \mathrm{~L}\right]^{6+}$ species. The addition of the two protons results quite favorable with constants ranging from 6.96 to 4.39
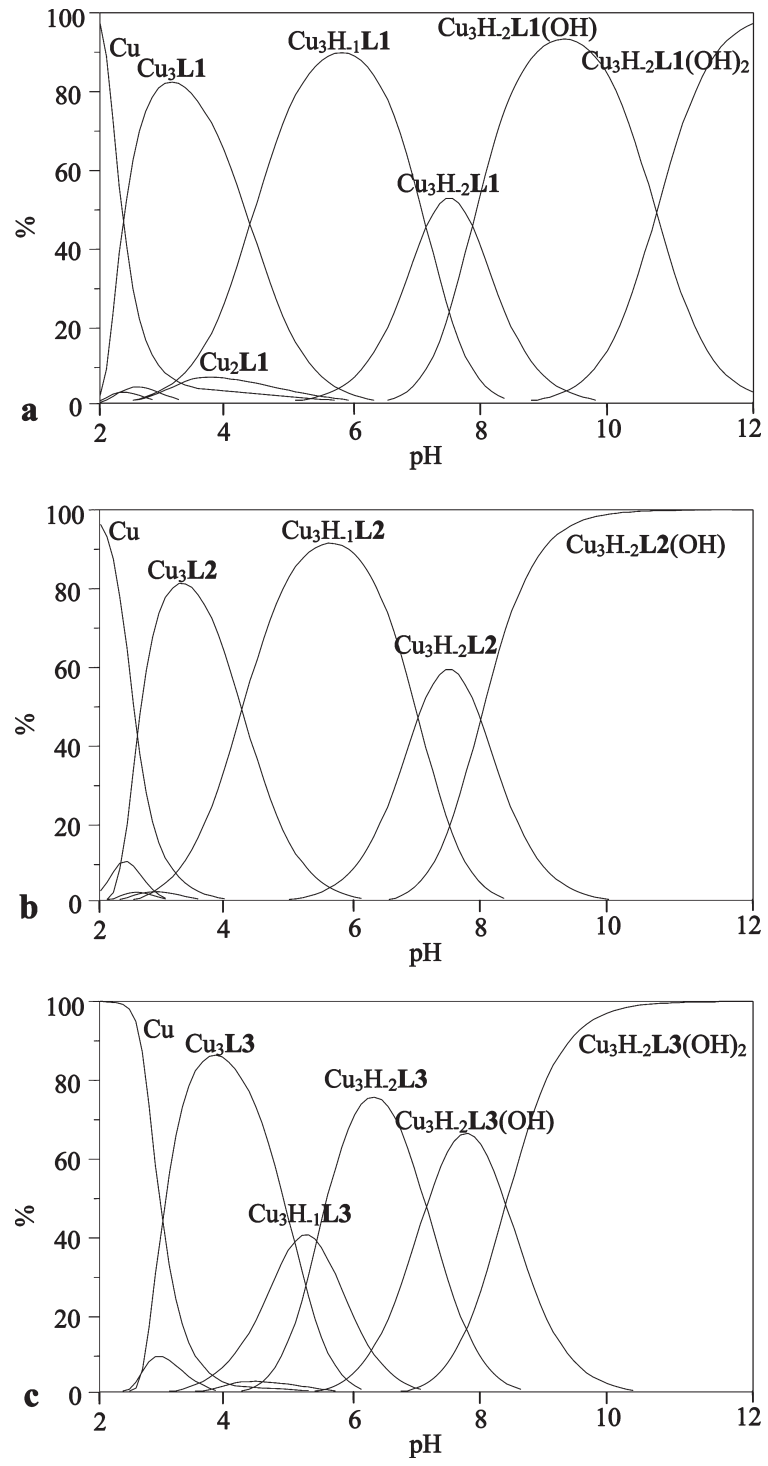

Figure 5. Distribution diagrams of the species for the $\mathrm{L} / \mathrm{Cu}$ (II) systems as a function of $\mathrm{pH}$ in aqueous solution $I=0.15 \mathrm{M} \mathrm{NMe}_{4} \mathrm{Cl}, T=298.1$ $\mathrm{K}$. $\mathrm{L}=\mathbf{L 1}(\mathrm{a}), \mathrm{L}=\mathbf{L 2}(\mathrm{b})$, and $\mathrm{L}=\mathbf{L} 3(\mathrm{c}) ;[\mathrm{L}]=1 \times 10^{-3} \mathrm{M},[\mathrm{Cu}(\mathrm{II})]=$ $3 \times 10^{-3} \mathrm{M}$

logarithmic units (see Table 5); this could be ascribed to the protonation of the two noncoordinated benzyl nitrogen atoms, one for each $\mathbf{L} \mathbf{4}$ subunit. In other words, the two amine benzyl functions, which are not involved in the coordination in the dinuclear complexes (Figures 1 and $4 \mathrm{c}$ ), probably are not coordinated in the trinuclear $\left[\mathrm{Cu}_{3} \mathrm{H}_{-2} \mathrm{~L}\right]^{4+}$ species as well. Merging these considerations with the disposition of the donor groups in the dinuclear species and considering the capability of the phenolate oxygen atom to bind two metal ions in a bridge disposition, we can suppose that the third $\mathrm{Cu}$ (II) is coordinated in the area located between the other two $\mathrm{Cu}(\mathrm{II})$; in this way, it is stabilized by the two phenolate oxygen atoms as well as some amine groups of the linear polyamine chain. A schematic drawing of this suggested coordination is reported in Figure 4d; each phenolate oxygen atom bridges two metal ions and the central $\mathrm{Cu}$ (II) ion bridges the two phenolate oxygen atoms forming a $\mathrm{Cu}-\mathrm{O}-\mathrm{Cu}-\mathrm{O}-\mathrm{Cu}$ sequence where the three metal ions are close to each other. 
The $\left[\mathrm{Cu}_{3} \mathrm{H}_{-2} \mathrm{~L}\right]^{4+}$ species undergoes further deprotonation processes highlighting that water molecules are coordinated to the metal centers. In this case, reporting the reaction as hydroxide addition to the trinuclear species, the process is favorable to the formation of the hydroxylated $\left[\mathrm{Cu}_{3} \mathrm{H}_{-2} \mathrm{LOH}\right]^{3+}$ species (Table 5). The values of the constants for the first hydroxide addition are high $(\log K=6.01,6.00$, and 6.64 for $\mathbf{L 1}, \mathbf{L 2}$, and $\mathbf{L 3}$, respectively) suggesting a bridged coordination of the $\mathrm{OH}^{-}$between two $\mathrm{Cu}(\mathrm{II})$ ions. Only the $\mathbf{L 1}$ and $\mathbf{L 3}$ complexes are able to add a second $\mathrm{OH}^{-}$, most probably in a nonbridged coordination mode.

Concluding, this family of ligands behaves similarly in $\mathrm{Cu}(\mathrm{II})$ coordination, forming stable mono-, di-, and trinuclear species depending on the used ligand to metal molar ratio; the mononuclear species are predominant for $\mathrm{L}: \mathrm{Cu}(\mathrm{II})$ molar ratio of $1: 1$ and the dinuclear for a ratio of $1: 2$, while the trinuclear are predominant in a 1:3 molar ratio; this highlights the high versatility of these ligands and their ability to form polynuclear complexes in aqueous solution.

\section{Concluding Remarks}

The three synthesized molecules form a new family of polyamino phenolic ligands showing similar acid-base and coordination properties toward the $\mathrm{Cu}(\mathrm{II})$ ion. As concerning the acid-base properties, they are strong polyprotic bases able to achieve the octaprotonated $\mathrm{H}_{8} \mathrm{~L}^{8+}$ species. They are able to strongly bind $\mathrm{Cu}$ (II) forming polynuclear species ranging from the mono- to the trinuclear type, depending on the used ligand to metal molar ratio; the mono-, di-, or trinuclear species prevail over the others highlighting the high stability of each species formed. A dinuclear species was isolated in the solid state and analyzed by X-ray crystallography affording the structure of the dinuclear $\left[\mathrm{Cu}_{2} \mathbf{L} 3\right]^{4+}$ cation; each coordinated $\mathrm{Cu}(\mathrm{II})$ ion is stabilized by a half of the molecule, and it draws the metals close to each other (about $5.3 \AA$ ). Each metal is stabilized by one of the macrocyclic subunits and by some nitrogen atoms provided by the linear polyamine chain; this scheme also seems to be preserved in the mononuclear species; that is, the first $\mathrm{Cu}(\mathrm{II})$ is stabilized by half of the ligand, while the other remains uninvolved in the coordination and thus can undergo the protonation. The trinuclear species are probably the most interesting species, and the involvement in a bridge disposition between two $\mathrm{Cu}$ (II) ions of both phenolate oxygen atoms can be suggested; all the ligands are able to assemble three $\mathrm{Cu}(\mathrm{II})$ forming highly charged and preorganized polynuclear systems. The studies highlight that both dinuclear and trinuclear complexes are able to add secondary ligands such as $\mathrm{OH}^{-}$, and in some cases, two $\mathrm{Cu}$ (II) can cooperate in stabilizing the guest by coordinating it in a bridged disposition. This makes these species attractive for several uses ranging from molecular recognition to transport and catalysis studies. In particular, they are interesting for studies involving polynuclear $\mathrm{Cu}(\mathrm{II})$ metal centers, placed at short distance, such as in the mimicking of the active sites of several multicopper enzymes.

Supporting Information Available: Listings of tables of crystallographic data, positional parameters, isotropic and anisotropic thermal factors, bond distances, and angles in CIF format. Distribution diagrams of the species as a function of $\mathrm{pH}$ for the $\mathrm{L} / \mathrm{Cu}$ (II) systems for $\mathrm{L}$ to $\mathrm{Cu}(\mathrm{II}) 1: 1$ and 1:2 molar ratios. This material is available free of charge via the Internet at http://pubs.acs.org.

Acknowledgment. The authors thank the Italian Ministero dell'Istruzione dell'Università e della Ricerca (MIUR), PRIN2007, for financial support. X-ray data collection was performed at CRIST (Centro Interdipartimentale di Crystallografia Strutturale), University of Florence. 